\title{
Auf dem Weg zu einer globalen Sozialgeschichte? Neuere Studien zur Globalgeschichte des Bürger- tums
}

Towards a global social history? New studies on the global history of the middle classes

\begin{abstract}
This contribution presents an overview of the numerous studies on the history of non-European middle classes which have been published within the last few years. It argues that the establishing of middle classes in different parts of the world can be construed to a considerable extent as the result of global entanglements in the long nineteenth century. The article further discusses the epistemological problems, however also the advantages, of describing these social groups which emerged in the non-European world in the age of empire with European concepts such as bourgeoisie or middle class. Moreover, it explores whether an examination of their history could be extended to a global social history in a next step.
\end{abstract}

Globalgeschichtliche und postkoloniale Ansätze haben die Geschichtswissenschaft in einer Art und Weise verändert, die vor einigen Jahren noch undenkbar erschien.' Studien zu Kolonialphantasien und globalen entanglements füllen inzwischen ganze Bücherregale und sind definitiv im Mainstream der Disziplin angekommen. Allerdings fällt bei einem Blick auf den aktuellen output der Globalgeschichtsschreibung eine eigentümliche Leerstelle auf. Der Begriff der ,Gesellschaft', noch vor zwei Jahrzehnten die Basis jeder theoriegeleiteten Geschichtsschreibung, taucht kaum mehr auf. Stattdessen interessiert sich die globalhistorische Forschung vor allem für imperiale Expansionen, koloniale Phantasmen oder das Knüpfen von Netzwerken, die aber oft nicht mehr explizit sozial verortet werden. ${ }^{1}$ Jürgen Osterhammel hat deshalb schon vor längerem den Umstand problematisiert, dass globale und transnationale Ansätze über "kein sozialstrukturelles Substrat" verfügten. ${ }^{2}$ Auch Koryphäen der amerikanischen world history wie Patrick Manning und Kenneth Pomeranz haben die Vernachlässigung des Sozialen beklagt. Kenneth Pomeranz hat dabei die Ansicht vertreten, „world history has much to gain from developing research agendas with a strong social history component and from thinking of social history in broad terms ${ }^{\text {"3 }}$. Doch weder er

- Ich danke Valeska Huber und David Motadel für ihre Kommentare zu einer früheren Version dieses Beitrages.

1 So werden weder in Chris Baylys monumentalen Globalgeschichte des 19. Jahrhunderts noch in Jane Burbanks und Frederick Coopers Imperialismus-Studie oder den ersten beiden Bänden der auf sechs Bände angelegten Weltgeschichte von Harvard UP und Beck spezifische Überlegungen zu einer globalen Gesellschaftsgeschichte angestellt: Bayly, Christopher A.: Die Geburt der modernen Welt. Eine Globalgeschichte 1780-1914, Campus, Frankfurt a. M./New York 2006; Burbank, Jane/Cooper, Frederick: Empires in World History. Power and the Politics of Difference, Princeton UP, Princeton, NJ 2010; Rosenberg, Emily S. (Hrsg.): A World Connecting, 1870-1945, Harvard UP, Cambridge, MA/London 2012; Iriye, Akira (Hrsg.): Global Interdependence. The World After 1945, Harvard UP, Cambridge, MA London 2014. Eine Ausnahme stellt Jürgen Osterhammels Studie zum 19. Jahrhundert dar, die ein längeres Kapitel zu sozialen Hierarchien beinhaltet: Osterhammel, Jürgen: Die Verwandlung der Welt. Eine Geschichte des 19. Jahrhunderts, Beck, München 2009.

2 Osterhammel, Jürgen: Transnationale Gesellschaftsgeschichte. Erweiterung oder Alternative?, in: Geschichte und Gesellschaft 27 (2001), H. 3, S. 464-479, hier S. 475.

3 Pomeranz, Kenneth: Social History and World History. From Daily Life to Patterns of Change, in: Journal of World History 18 (2007), H. 1, S. 69-98, hier S. 70. 
noch Manning kommen über allgemeine Anregungen hinaus, wie eine Verbindung zwischen Global- und Sozialgeschichte allenfalls bewerkstelligt werden könnte. ${ }^{4}$

Dies hat nicht zuletzt damit zu tun, dass sich eine Weltgesellschaft, die mit nationalen Gesellschaften vergleichbar ist, analytisch nur schwer konzipieren lässt. Während die Sozialgeschichte den Nationalstaat gewissermaßen als historiographisches Laboratorium verwenden kann, um wirtschaftliche Entwicklungen, Institutionenbildung, politische Machtkämpfe und soziale Schichtungsprozesse zu beobachten, fehlt der Globalgeschichte ein solch eindeutig fassbarer Raum, um das Wechselspiel derartiger gesellschaftlicher Prozesse zu beobachten. ${ }^{5}$ Der Reiz und das analytische Potenzial der Globalgeschichte bestehen im Gegenteil gerade darin, Verflechtungen über bestehende territoriale Grenzen zu verfolgen und dabei die Inkommensurabilität von staatlichen Ordnungsversuchen, ökonomischen Marktstrukturen, kulturellen Deutungshorizonten und sozialen Wanderungsbewegungen feststellen zu können. ${ }^{6}$

Trotz dieses Mankos sind in den letzten Jahren verschiedene Studien aus dem Bereich der globalen und außereuropäischen Geschichte erschienen, die in ihrem Kern sozialhistorisch argumentieren. Dies betrifft in erster Linie das ständig wachsende Feld der global labour history. ${ }^{7}$ Darüber hinaus erschienen jedoch auch zahlreiche Untersuchungen, die jene Mittelschichten thematisieren, die sich ab dem 19. Jahrhundert in Afrika, Asien, Lateinamerika und dem Nahen Osten herausgebildet haben, und die in vielerlei Hinsicht mit dem europäischen Bürgertum verglichen werden können. Ihre bloße Existenz stellt die lange Zeit von der europäischen Sozialgeschichte vertretene Gewissheit in Frage, es habe im 19. und frühen 20. Jahrhundert außerhalb des nordatlantischen Raumes keine gesellschaftliche Formation gegeben, die mit dem westlichen Bürgertum vergleichbar sei ${ }^{8}$ - und diese Studien erscheinen wohl nicht zufällig zu einem Zeitpunkt, zu dem in Asien, Lateinamerika und dem Nahen Osten aufgrund des wirtschaftlichen Aufschwungs große Teile der Bevölkerung in die Mittelschicht aufgestiegen sind. ${ }^{9}$ In Bezug auf die europäische Geschichte wiederum hat die new imperial history aufgezeigt, dass auch hier der "bürgerliche Wertehimmel" 10 einen durchaus globalen Horizont besaß. Wirtschaftsbürgerliche Eliten waren häufig weltweit vernetzt ${ }^{11}$; und die in erster Linie vom europäischen

4 Manning, Patrick: Navigating World History. Historians Create a Global Past, Palgrave Macmillan, Basingstoke/New York 2003, S. 201-213.

5 Nach wie vor beispielhaft für die Konzipierung einer derartigen historischen Sozialwissenschaft: Wehler, Hans-Ulrich: Deutsche Gesellschaftsgeschichte, 5 Bde., Beck, München 1987-2008.

6 Siehe hierzu etwa Conrad, Sebastian/Eckert, Andreas/Freitag, Ulrike (Hrsg.): Globalgeschichte. Theorien, Ansätze, Themen, Campus, Frankfurt a. M./New York 2007.

7 Linden, Marcel van der: Workers of the World. Essays Toward a Global Labor History, Brill, Leiden/ Boston 2008; s. a. das dritte Kapitel von Neuheiser, Jörg: Arbeit zwischen Entgrenzung und Konsum. Die Geschichte der Arbeit im 20. Jahrhundert als Gegenstand aktueller zeithistorischer und sozialwissenschaftlicher Studien, in: Neue Politische Literatur 58 (2013), H. 3, S. 421-448.

8 Siegrist, Hannes: Bourgeoisie/Middle Classes, History of, in: Smelser, Neil J./Baltes, Paul B. (Hrsg.): International Encyclopedia of the Social \& Behavioral Sciences, Elsevier, Amsterdam u. a. 2001, S. 1.307-1.314, hier S. 1.312 .

9 Laut dem indischen Ökonomen Surjit Bhalla können heute über die Hälfte der Weltbevölkerung zur Mittelklasse gerechnet werden: Parker, John: Burgeoning Bourgeoisie, in: The Economist, 12. Februar 2009, URL: <http://mww.economist.com/node/13063298> [Zugriff: 20.05.2014].

10 Hettling, Manfred/Hoffmann, Stefan-Ludwig (Hrsg.): Der bürgerliche Wertehimmel. Innenansichten des 19. Jahrhunderts, Vandenhock \& Ruprecht, Göttingen 2000.

11 Dejung, Christof: Die Fäden des globalen Marktes. Eine Sozial- und Kulturgeschichte des Welthandels am Beispiel der Handelsfirma Gebrüder Volkart 1851-1999, Böhlau, Köln u. a. 2013. 
Bildungsbürgertum getragene metropolitane Wissenschaft begründete ihren universellen Anspruch, indem sie Pflanzen, Tiere und menschliche Zivilisationen aus aller Welt miteinander verglich. Zudem beruhte die bürgerliche Kultur auch auf dem Konsum von ,Kolonialwaren' wie Tee, Kaffee, Kakao oder Seide oder der kontemplativen Belehrung über exotische Tiere und Pflanzen durch den Besuch zoologischer und botanischer Gärten. ${ }^{12}$ Durch eine derartige Rekontextualisierung des europäischen und nichteuropäischen Bürgertums eröffnet sich eine Untersuchungsperspektive, die sozialhistorische und globalgeschichtliche Ansätze miteinander verbinden kann. Die Herausbildung von bürgerlichen Mittelklassen in verschiedenen Teilen der Welt könnte als eine Folge globaler Vernetzungen interpretiert werden; umgekehrt gehörten Mittelklassen ab dem 19. Jahrhundert zu den wichtigsten sozialen Gruppen, welche die genannten wirtschaftlichen, kulturellen und wissenschaftlichen Netze knüpften. ${ }^{13}$

Doch ist es wirklich statthaft, soziale Gruppen in verschiedenen Teilen der Welt mit aus dem europäischen Kontext stammenden Konzepten wie Bürgertum oder Mittelklasse zu benennen? Immerhin bezeichneten sich die indischen ashrafs, die afrikanischen Eliten ${ }^{14}$ oder die ägyptischen effendis nicht in jedem Fall explizit als middle class oder Teil einer bourgeoisie. Zudem war das 19. Jahrhundert nicht nur das goldene Zeitalter des Bürgertums, sondern auch das Zeitalter des Imperialismus. Scheinbar universelle Konzepte wie jenes der Mittelklasse auf außereuropäische Gesellschaften anzuwenden - so geben postkoloniale Theoretiker wie Dipesh Chakrabarty zu bedenken -, habe zur Folge, dass diese gegenüber der als idealtypisch angesehenen europäischen Gesellschaft von vornherein als defizitär gälten. Damit wiederum werde die angebliche zivilisatorische Überlegenheit des Westens bekräftigt. ${ }^{15} \mathrm{Da}$ es keine indigenen Gegenmodelle gibt, kann man dem Problem, das sich aus der epistemologischen Hegemonie westlicher Analysekonzepte ergibt, nur schwer entgehen. Ein vollständiger Verzicht auf interkulturelle Vergleiche mittels bestimmter konzeptioneller Grundlagen würde aber letzten Endes die - von der postkolonialen Theorie ebenfalls beklagte - Vorstellung bekräftigen, die nichtwestlichen Gesellschaften seien so ganz , anders' als die westlichen und so einer wenig produktiven Exotisierung Vorschub leisten. Das Dilemma lässt sich nicht auflösen, aber immerhin abschwächen und für empirische Untersuchungen nutzbar machen, wenn man nicht in der Dichotomie von Differenzen und Identitäten stecken bleibt, sondern der Anregung Anil Bhattis folgt, nach Ähnlichkeiten zu fragen, um ein plurikulturelles Narrativ von Modernität zu entwickeln. ${ }^{16}$ Dies erlaubt eine Verständigung über geographische Grenzen hinweg und verhindert den intellektuellen Verlust,

12 Drayton, Richard: Nature's Government. Science, British Imperialism and the Improvement of the World, Yale UP, New Haven, CT/London 2000; Menninger, Annerose: Genuss im kulturellen Wandel. Tabak, Kaffee, Tee und Schokolade in Europa (16.-19. Jahrhundert), Steiner, Stuttgart 2004; Hall, Catherine/ Rose, Sonya O. (Hrsg.): At Home with the Empire. Metropolitan Culture and the Imperial World, Cambridge UP, Cambridge 2006.

13 In konzeptioneller Hinsicht kann dieser Vergesellschaftungsprozess mit der Strukturalisierungstheorie von Anthony Giddens interpretiert werden: Giddens, Anthony: The Constitution of Society. Outline of the Theory of Structuration, Polity, Cambridge 1984.

14 Vgl. hierzu etwa Doortmont, Michel R.: The Pen-Pictures of Modern Africans and African Celebrities by Charles Francis Hutchison. A Collective Biography of Elite Society in the Gold Coast Colony, Brill, Leiden/Boston 2005.

15 Chakrabarty, Dipesh: Provincializing Europe. Postcolonial Thought and Historical Difference, Princeton UP, Princeton, NJ 2000.

16 Bhatti, Anil u. a.: Ähnlichkeit. Ein kulturtheoretisches Paradigma, in: Internationales Archiv für Sozialgeschichte der deutschen Literatur 36 (2011), H. 1, S. 233-247. 
der durch einen vollständigen Rückzug von europäischer und außereuropäischer Geschichte in ihre jeweiligen areas entstehen würde. ${ }^{17}$

Gerade ein globalhistorischer Zugang, der die Entstehung bürgerlicher Mittelklassen als Resultat eines weltweiten Vergesellschaftungsprozesses sieht, kann von einer derartigen Suche nach Ähnlichkeiten profitieren. In der Sozialgeschichte ist die These, wonach soziale Klassen nicht einfach als Funktion bestimmter ökonomischer Parameter seien, sondern durch soziale Praktiken reproduziert werden, inzwischen breit akzeptiert. ${ }^{18}$ Für die Untersuchung der europäischen Mittelklassen hat sich als hilfreich erwiesen, davon auszugehen, dass die Zugehörigkeit zu dieser Schicht eine bestimmte Art der Lebensführung voraussetzte - eine bestimmte Art sich zu kleiden, zu essen, sich zu bewegen, seine Emotionen zu kontrollieren sowie das Bemühen zur Ausbildung einer individuellen Persönlichkeit, weiter den Glauben an Fortschritt und Rationalität, eine bestimmte Arbeitsmoral und die Eingliederung in eine dichotome Geschlechterordnung. ${ }^{19}$ Diese bürgerliche Kultur konnte sich jedoch nicht unabhängig von ökonomischen Rahmenbedingungen ausbilden; im Gegenteil, die Fähigkeit kulturelles Kapital zu erwerben, setzte einen einigermaßen gesicherten ökonomischen Status voraus.

Die im folgenden Beitrag vorgestellten Studien kreisen alle um die Frage, ob sich derartige kulturelle Merkmale auch bei außereuropäischen Mittelschichten feststellen lassen. Hatten Anwälte in Kairo, Journalisten in Tokio, Kaufleute in Shanghai, Archäologen in Cuzco oder Ärzte in Delhi ähnliche Vorstellungen davon, wie sie ihr Leben gestalten wollten wie Bankiers in Antwerpen oder Hochschullehrer in Berlin? Gibt es Ähnlichkeiten in der Art, wie sie sich kleideten ${ }^{20}$, wie die Beziehungen zwischen Männern und Frauen organisiert wurden und wie sie über die jeweiligen sozialen Unterschichten dachten? Wenn dies der Fall sein sollte und wenn sich zudem die Vermutung bewahrheitet, dass diese Mittelschichten ihre soziale Position auch dadurch zu festigen suchten, indem sie sich in Bezug zu sozialen Prozessen in anderen Teilen der Welt setzten, dann könnte dies ein erster Schritt zu einer Globalgeschichte der Mittelklassen sein, die sich in einem weiteren Schritt allenfalls zu einer globalen Sozialgeschichte enweitern ließe.

\section{Auf der Suche nach der globalen Mittelklasse}

Wie wenig haltbar die Vorstellung ist, die bürgerlichen Mittelklassen seien ein Phänomen, das sich nur in Westeuropa und den USA feststellen lässt, zeigen A. Ricardo López und Barbara Weinstein mit ihrem Sammelband zur transnationalen Geschichte der Mittelklasse. ${ }^{21}$

17 Ein nur teilweise geglückter, weil mehrheitlich innerhalb einzelner nationalhistorischer Narrative stehen bleibender Versuch für einen solchen Brückenschlag aus dem Bereich der Bürgertumsforschung ist Amhmad, Imtiaz/Reifeld, Helmut (Hrsg.): Middle Class Values in India \& Western Europe, Social Science Press, New Delhi 2003.

18 Thompson, Edward P.: The Making of the English Working Class, Gollancz, London 1963.

19 Die kulturelle Ähnlichkeit zwischen den Mittelklassen in Großbritannien, Australien und den USA wird exemplarisch dargestellt in Young, Linda: Middle-Class Culture in the Nineteenth Century, Palgrave Macmillan, Basingstoke/New York 2002. Nach wie vor maßgebend für die kulturtheoretische Fundierung von sozialen Differenzen ist Bourdieu, Pierre: Die feinen Unterschiede. Kritik der gesellschaftlichen Urteilskraft, Suhrkamp, Frankfurt a. M. 2007.

20 Ross, Robert: Clothing. A Global History. Or, the Imperialists' New Clothes, Polity Press, Cambridge 2008.

21 López, A. Ricardo/Weinstein, Barbara (Hrsg.): The Making of the Middle Class. Toward a Transnational History, Duke UP, Durham, NC/London 2012. 
In den Beiträgen wird exemplarisch aufgezeigt, wie an so unterschiedlichen Orten wie Indien, Peru oder dem subsaharischen Afrika ab Mitte des 19. Jahrhunderts die Herausbildung einer Gesellschaftsformation beobachtet werden kann, die mit der euro-amerikanischen Mittelklasse vergleichbar ist. Dabei sind sich die Herausgeber der Schwierigkeiten durchaus bewusst, die der Versuch mit sich bringt, eine weltweit gültige Definition von Mittelklasse entwickeln zu wollen. Anders als die Aristokratie oder die Arbeiterklasse verfügte die bürgerliche Mittelschicht weder über herkunftsbedingte Privilegien oder ein klares politisches Programm, noch ließ sie sich durch bestimmte sozioökonomische Parameter klar von anderen Sozialformationen abgrenzen. Verschiedene Autorinnen und Autoren haben deshalb die Ansicht vertreten, die Mittelklasse sei aufgrund ihres amorphen Charakters eine untaugliche Kategorie für die historische Analyse. ${ }^{22}$ Dieses Problem potenziert sich bei einer globalhistorischen Ausweitung des Erkenntnisinteresses. López und Weinstein argumentieren jedoch plausibel, dass sich historische Akteure ab dem beginnenden 19. Jahrhundert in ganz verschiedenen Teilen der Welt bestimmter Diskurse bedienten und sich durch vergleichbare soziale Praktiken von der übrigen Gesellschaft abzugrenzen suchten. Diese Diskurse und Praktiken seien hinreichend ähnlich, um die These vertreten zu können, „that [they] were facets of a single - yet complex and variegated - historical formation occurring over most of the globe from the nineteenth century on ${ }^{\text {"23 }}$.

Die Tragfähigkeit dieses Ansatzes soll im Folgenden an drei Beiträgen des Sammelbandes exemplarisch verdeutlicht werden. So zeigt etwa Micheal O. West, wie sich in Südrhodesien Ende des 19. Jahrhunderts eine afrikanische Mittelklasse herauszubilden begann, die im kolonialen Masterplan nicht vorgesehen war. ${ }^{24}$ Weder die christlichen Missionare - in deren Schulen die Afrikaner eine westliche Bildung genossen - noch die koloniale Administration hatten ursprünglich ein Interesse an der Ausbildung einer einheimischen Elite in der Siedlerkolonie. Ohne eine solche Elite konnten viele koloniale Aspirationen jedoch nicht verwirklicht werden; die Missionsschulen waren auf afrikanische Lehrer angewiesen und die koloniale Bürokratie und die europäischen Unternehmen benötigten einheimische Angestellte, um funktionieren zu können. Und schon bald wurden auch afrikanische Ärzte, Krankenschwestern und Sozialarbeiter für die Aufrechterhaltung der sozialen Ordnung wichtig. Aus dieser wachsenden afrikanischen Mittelklasse rekrutierten sich insbesondere nach dem Ersten Weltkrieg die Aktivisten der Unabhängigkeitsbewegung, welche die weiße . Kolonialherrschaft immer stärker in Frage stellten. ${ }^{25}$

In einem weiteren Beitrag zeigt Prashant Kidambi, wie sich in Bombay Ende des 19. Jahrhunderts eine englisch gebildete intermediäre Gruppe von Anwälten, Ärzten, Ingenieuren, Geschäftsleuten, Journalisten, Lehrern und Anwälten herausbildete, die sich selber explizit als middle class bezeichnete. ${ }^{26}$ Die Angehörigen dieser Gesellschaftsformation stammten einerseits aus schriftkundigen Kasten wie den Brahmanen und den Pathare Prabhus, und andererseits aus der indischen Wirtschaftselite, etwa der Gruppe der parsischen Kaufleute.

22 Maza, Sarah: The Myth of the French Bourgeoisie. An Essay on the Social Imaginary, 1750-1850, Harvard UP, Cambridge, MA/London 2003, S. 4.

23 López, A. Ricardo/Weinstein, Barbara: We Shall Be All. Toward a Transnational History of the Middle Class, in: López/Weinstein: Middle Class (wie Anm. 21), S. 1-25, hier S. 19.

24 West, Michael O.: The African Middle Class in Zimbabwe. Historical and Contemporary Perspectives, in: ebd., S. 45-57.

25 Vgl. zum afrikanischen Kontext die bereits ältere Studie Kuper, Leo: An African Bourgeoisie. Race, Class, and Politics in South Africa, Yale UP, New Haven, CT/London 1965.

26 Kidamdi, Prashant: Colonial Bombay, 1890-1940, in: López/Weinstein: Middle Class (wie Anm. 21), S. 141-160. 
Sie orientierten sich an westlichen Vorstellungen von politischer Öffentlichkeit, Hygiene und einem disziplinierten Umgang mit dem Selbst und begannen ab der Jahrhundertwende karitative Organisationen wie etwa die Social Service League zu gründen. Diese Organisationen hatten das Ziel, die soziale Situation der indischen Armen zu verbessern, von denen sich die Angehörigen der indischen Mittelschicht klar abgrenzten und deren Umgangsformen sie ablehnten. Keith David Watenpaugh schließlich zeigt, wie sich im Nahen Osten zu Beginn des 20. Jahrhunderts aus einer sehr heterogenen Gruppe von Kaufleuten, Landbesitzern und muslimischen wie nicht-muslimischen Gelehrten ein arabisches Bürgertum herausbildete. ${ }^{27}$ Dieses war zwar fähig, zivilgesellschaftliche Institutionen einzurichten, konnte diese Formen der Vergesellschaftung aber nicht in wirkliche politische Macht ummünzen. Watenpaugh bestätigt dabei die Resultate früherer Studien ${ }^{28}$, wonach nicht zuletzt die Differenzen zwischen Muslimen und den Angehörigen religiöser Minderheiten eine grundsätzliche Modernisierung der Gesellschaft verhinderten. Während etwa die mehrheitlich katholischen Mitglieder der Aleppo Mutual Aid Society der Ansicht waren, nur ein säkularisierter Islam könne die Grundlage für eine gesellschaftliche Modernisierung sein, beharrten Angehörige der muslimischen Mittelschicht darauf, dass der Islam auch in einer erneuerten Gesellschaft als Staatsreligion bestehen bleiben sollte, weshalb der Reformeifer der Mittelklassen ab Beginn des 20. Jahrhunderts ins Stocken geriet.

Der Band von López und Weinstein macht deutlich, dass sich die außereuropäischen Mittelklassen in der Regel aus Gruppen wie Gelehrten, Kaufleuten oder Landbesitzern rekrutierten, die bereits vor dem 19. Jahrhundert eine intermediäre Position zwischen dem lokalen Adel und den jeweiligen Unterschichten eingenommen hatten. Wie auch in Europa zeigt sich beim außereuropäischen Bürgertum eine latente Spannung zwischen universalistischen Gleichheitsidealen einerseits und dem steten Bemühen, sich von den urbanen und ruralen Unterschichten abzugrenzen, andererseits. Indem die lokalen Mittelschichten in koIonialen Besitzungen in eine widersprüchliche Position zum europäischen Modernisierungsprojekt gerieten, wurde ihre soziale Stellung zusätzlich kompliziert. Zum einen legitimierten die Mittelklassen in Asien, Afrika und dem Nahen Osten ihre soziale Stellung durch einen Rückgriff auf kulturelle Konzepte, die sie aus dem Westen übernommen hatten. Zum anderen schränkte die Kolonialherrschaft die politischen Handlungsspielräume der jeweiligen lokalen Mittelklassen stark ein, die sich zudem durch den kolonialen Rassismus stets in einer inferioren Position gegenüber der europäischen Zivilisation befanden. Der Sammelband liefert damit aufgrund seiner empirisch gesättigten Beiträge, die oftmals auf bereits zuvor publizierten Monographien beruhen ${ }^{29}$, wertvolle Anhaltspunkte für eine Globalgeschichte des Bürgertums. Dennoch weist er konzeptionell und theoretisch einige Schwachpunkte auf. So ist der Band überaus breit angelegt, und umfasst neben Beiträgen zu kolonialen Mittelklassen und den Mittelklassen in Lateinamerika - auf die weiter unten noch näher eingegangen wird - auch Aufsätze zur deutschen Frauenbewegung, der amerikanischen

27 Watenpaugh, Keith David: Being Middle Class and Being Arab. Sectarian Dilemmas and Middle-Class Modernity in the Arab Middle East, 1908-1936, in: López/Weinstein: Middle Class (wie Anm. 21), S. 267-287.

28 Göçek, Müge Fatma: Rise of the Bourgeoisie, Demise of Empire. Ottoman Westernization and Social Change, Oxford UP, Oxford u. a. 1996.

29 So etwa für die angeführten Beiträge: West, Michael O.: The Rise of the African Middle Class. Colonial Zimbabwe, 1898-1965, Indiana UP, Bloomington/Indianapolis, IN 2002; Watenpaugh, Keith David: Being Modern in the Middle East. Revolution, Nationalism, Colonialism, and the Arab Middle Class, Princeton UP, Princeton, NJ/Oxford 2006.

\section{4}


folk-Bewegung und der Immigration nach Kanada während des Kalten Krieges, die mit den oben angeführten und eher globalhistorisch beziehungsweise (post)kolonial angelegten Aufsätzen kaum etwas gemein haben. Zudem wird das Postulat der Verflechtungsgeschichte, wonach sich historische Prozesse durch eine Analyse der globalen Transfers von Menschen, Gütern und Ideen erklären ließen ${ }^{30}$, zwar verschiedentlich erwähnt, aber nur in einem Teil der Beiträge wirklich empirisch umgesetzt. Gerade die Beiträge zu Europa und Nordamerika kommen ohne jede Untersuchung der Rückwirkungen des Kolonialismus auf die westliche Gesellschaft aus, womit eine zentrale Forderung der postkolonialen Theorie und der neueren Imperialgeschichte nicht berücksichtigt wird.

\section{Indien: die Mittelklasse als Produkt eines derivativen Diskurses?}

Von allen nichtwestlichen Ländern hat sicherlich Indien die längste Tradition in der Erforschung der Geschichte des Bürgertums. Ab den 1980er Jahren problematisierten die Vertreterinnen und Vertreter der subaltern studies die politische Unabhängigkeit Indiens als Projekt einer westlich gebildeten Bourgeoisie. ${ }^{31}$ Es wurde insbesondere vorgebracht, dass die indische Mittelklasse ihre Vorherrschaft innerhalb der indischen Gesellschaft aus einer Position der kolonialen Subalternität heraus entwickelt habe. So verwies Partha Chatterjee darauf, dass diese Gruppe ihr Selbstverständnis in einer medialen Öffentlichkeit ausgebildet habe, in der auf Englisch kommuniziert wurde - während die Verkehrssprache der indischen Eliten bis Anfang des 19. Jahrhunderts persisch war. Chatterjee argumentierte deshalb, die soziale Identität des indischen Bürgertums habe mehrheitlich auf einer Imitation westlicher Vorbilder beruht und müsse deshalb als „derivativer Diskus“32 angesehen werden.

Gegen diese Ansicht wurde jedoch verschiedentlich Einspruch erhoben. Laut Sanjay Joshi kann die Herausbildung einer indischen Mittelklasse nicht einfach als Übernahme westlicher Modernitätskonzepte gesehen werden. Insbesondere verkenne eine solche Ansicht die agency dieser sozialen Schicht, die danach strebte, sich sowohl nach oben (gegen die indische Aristokratie und die britischen Kolonisatoren) wie nach unten (gegen die indischen Unterschichten) abzugrenzen. Nach Joshis Ansicht tat sie dies, indem sie sich auf vorkoloniale Vorstellungen politischer und sozialer Organisation stützten. Dabei sei es - ähnlich wie in Europa - zu einem Konflikt zwischen universalistischen Freiheitsideen und dem Bemühen, soziale Macht zu erringen, gekommen. Joshi verdeutlicht dies am Beispiel der Geschlechterbeziehungen: Indische Mittelklasseaktivisten hätten zwar gefordert, auch indische Frauen sollten eine Schulbildung genießen können. Gleichzeitig habe die Propagierung traditioneller Familienkonzepte im Mittelschichtsdiskurs aber auch zu einer stärkeren Disziplinierung der Frauen geführt, die sich gemäß diesen Konzepten ihren Ehemännern unterzuordnen hätten. ${ }^{33}$

30 Subrahmanyam, Sanjay: Connected Histories. Notes Towards a Reconfiguration of Early Modern Eurasia, 1400-1800, in: Modern Asian Studies 31 (1997), H. 3, S. 735-762; Bayly: Geburt (wie Anm. 1).

31 Sarkar, Sumit: ,Popular' Movements and ,Middle Class' Leadership in Late Colonial India. Perspectives and Problems of a ,History from Below', Bagchi Centre for Studies in Social Sciences, Calcutta 1983.

32 Chatterjee, Partha: Nationalist Thought and the Colonial World. A Derivative Discourse, Zed, London 1986; ders.: The Nation and Its Fragments. Colonial and Postcolonial Histories, Princeton UP, Princeton, NJ 1993.

33 Joshi, Sanjay: Fractured Modernity. Making of a Middle Class in Colonial North India, Oxford UP, New Delhi u. a. 2001. 
Gerade, weil die Geschichte der indischen Mittelklasse in den letzten Jahrzehnten äuBerst kontrovers diskutiert wurde, ist es zu begrüßen, dass Joshi in einem neueren Sammelband verschiedene bereits früher publizierte Texte von renommierten Spezialisten für die Geschichte des Subkontinents zugänglich macht. ${ }^{34}$ Neben einem Neuabdruck eines Kapitels aus Chatterjees "The Nation and its Fragments" findet sich auch ein Text von Christopher Bayly, in dem das Argument vertreten wird, eine politische Öffentlichkeit sei kein spezifisch westliches Konzept gewesen (wie etwa Jürgen Habermas postulierte ${ }^{35}$ ). Die indischen Nationalisten des 19. Jahrhunderts konnten vielmehr auf eine lange etablierte innerindische Tradition von Debatte und politischer Kommunikation zurückgreifen. ${ }^{36}$ Aufschlussreich ist schließlich der Beitrag von Claude Markovits, welcher die in vielen Studien vorgebrachte These hinterfragt, wonach die englisch gebildeten Angestellten der kolonialen Bürokratie das Zentrum der indischen Mittelklasse gewesen seien. ${ }^{37}$ Neben diesem indischen Bildungsbürgertum habe es auch noch eine kaufmännische Elite - mit anderen Worten: ein Wirtschaftsbürgertum - gegeben, das sich primär an kaufmännischen Ehrbegriffen orientierte, die sich im 18. Jahrhundert in Indien herausgebildet hatten. Zwar kam es zu Annäherungen zwischen indischen Bildungs- und Wirtschaftsbürgertum; ein wesentlicher Unterschied habe aber darin bestanden, dass die indischen Kaufleute über eine größere Autonomie vom Staat verfügten und im Notfall auf familiäre Netzwerke zurückgreifen konnten, während indische Angestellte, Beamte und Freiberufler wesentlich stärker vom Staat abhängig gewesen seien und sich deshalb auch aktiver für die nationale Unabhängigkeit eingesetzt hätten.

Wie gewinnbringend es sein kann, die äußerst heterogene Gruppe von Ärzten, Anwälten, Beamten und Kaufleuten als Angehörige eines indischen Bürgertums zu fassen, hat Margrit Pernau in ihrer eindrücklichen Studie zur muslimischen Mittelklasse Delhis im 19. Jahrhundert gezeigt, die inzwischen in einer englischen Übersetzung erschienen ist. ${ }^{38}$ Der Kern von Pernaus Untersuchung liegt in der Forderung, mit dem Konzept der Bürgerlichkeit eine analytische Kategorie zu entwickeln, die einerseits auf die spezifischen historischen Umstände im kolonialen Indien Rücksicht nimmt, und andererseits vermeidet, Indien als exotisches Wunderland zu charakterisieren, das nach ganz anderen Regeln funktionierte als der ,aufgeklärte' Westen. Nach Pernau verstanden sich die ashraf Delhis als eine Gruppe, deren Status im Wesentlichen durch ihre Respektabilität und ihre Bildung definiert wurde, und die nicht zuletzt durch das öffentliche Zelebrieren ihrer islamischen Religion zu einer sozialen Gemeinschaft wurden. Die Grenze zwischen den sozialen Gruppen, die im europäischen Kontext als Adel beziehungsweise als Mittelklasse definiert werden könnten, war in Nordindien äußerst durchlässig. Während bis Mitte des 19. Jahrhunderts nur Landbesitzer, Gelehrte, Geistliche, Beamte, Ärzte und Offiziere zu den ashraf gezählt wurden, erstreckte sich der Begriff nach den 1850er Jahren auch auf Kaufleute und Händler. Das hohe Ansehen

34 Ders. (Hrsg.): The Middle Class in Colonial India, Oxford UP, New Delhi u. a. 2010.

35 Jürgen Habermas ging von der Prämisse aus, dass bürgerliche Öffentlichkeit als sozialwissenschaftliche Kategorie ausschließlich für die Analyse der modernen westeuropäischen Gesellschaft geeignet sei: Habermas Jürgen: Strukturwandel der Öffentlichkeit. Untersuchungen zu einer Kategorie der bürgerlichen Gesellschaft, Suhrkamp, Frankfurt a. M. 1990 (orig. 1962), S. 51.

36 Erstabdruck in Bayly, Christopher A.: Empire and Information. Intelligence Gathering and Social Communication in India, 1780-1870, Cambridge UP, Cambridge 1996, S. 180-211.

37 Erstabdruck in Markovits, Claude: Merchants, Traders, Entrepreneurs. Indian Business in the Colonial Era, Palgrave Macmillan, Basingstoke/New York 2008, S. 167-83.

38 Pernau, Margrit: Ashraf into Middle Classes. Muslims in Nineteenth-Century Delhi, Oxford UP, New Delhi u. a. 2013 (orig. 2008).

\section{6}


ihrer Herkunft war zwar immer noch eines der zentralen Merkmale dieser Schicht, doch nun wurde die Bedeutung des beruflichen Erfolgs wesentlich stärker betont. Anders als in Europa strebten die muslimischen Mittelklassen Delhis jedoch nicht nach politischer Macht. Dies hat weniger mit kulturellen Spezifika des Islams zu tun, als vielmehr mit der kolonialen Situation und der Bündnispolitik der Briten; die ashraf hatten mehr zu gewinnen, wenn sie sich mit der Kolonialmacht arrangierten, als wenn sie selber politischen Einfluss eingefordert hätten. Alles in allem bestätigt Pernaus Untersuchung den Befund früherer Studien, wonach die Herausbildung einer indische Mittelklasse nicht erst für die letzten zwanzig Jahre und als Folge der ökonomischen Liberalisierung seit den 1990er Jahren zu beobachten ist. ${ }^{39}$ Die angeführten Untersuchungen zeigen vielmehr, dass sich die Entstehung eines indischen Bürgertums mit Gewinn bis in die Kolonialzeit zurückverfolgen lässt.

\section{Ambivalenzen statt clash of civilisations: osmanische und ägyptische Mittelschichten}

Viele der neueren Studien zur Herausbildung bürgerlicher Mittelschichten in kolonialen Gebieten betonen Momente der Ambivalenz und der Dialektik. Dies gilt auch für neuere Studien zum Osmanischen Rech und zu Nordafrika, was ein willkommener Gegensatz zum Klischee des clash of civilisations darstellt, demzufolge zwischen christlichem Westen und der islamischen Welt unvereinbare Gegensätze bestanden. ${ }^{40}$ Bruce Masters zeigt in seinem Übersichtswerk zur Geschichte der arabischen Bevölkerung im Osmanischen Reich, wie sich ab Mitte des 19. Jahrhunderts in Küstenstädten wie Izmir, Beirut und Haifa und später auch in syrischen Städten wie Damaskus und Aleppo sowie im Irak und auf der arabischen Halbinsel eine bourgeoisie herausbildete. Diese bestand aus Kaufleuten und Angestellten, Beamten, Lehrern, Ärzten und Anwälten, die sich für die politischen, wissenschaftlichen und technischen Neuerungen in Europa interessierten und versuchten, diese Entwicklungen auf das Osmanische Reich zu übertragen. ${ }^{41}$ Die neue Art der Lebensführung drückte sich unter anderem im Tragen westlicher Kleidung und neuen Wohnformen in Mehrfamilienhäusern aus, durch die sich die bürgerlichen Kleinfamilien räumlich von ihrer Verwandtschaft separieren konnten. Das Osmanische Reich reagierte auf den Reformwillen der neuen Mittelschichten durch infrastrukturelle Modernisierungen wie dem Bau von Straßenbahnen und Straßenbeleuchtung oder durch Gründung staatlicher Schulen. Für die muslimischen Modernisten bestand dabei - anders als für die europäischen Aufklärer - kein Widerspruch zwischen Religion und wissenschaftlichem Fortschritt; immerhin sei das ,goldene Zeitalter' des Islam ja mit einer wissenschaftlichen Hochblüte einhergegangen. ${ }^{42}$ Politischen Einfluss

39 Vgl. hierzu u. a. Fernandes, Leela: India's New Middle Class. Democratic Politics in an Era of Economic Reform, Minnesota UP, Minneapolis, MN/London 2006; Saavala, Minna: Middle-Class Moralities. Everyday Struggle over Belonging and Prestige in India, Orient BlackSwan, New Delhi 2010; Brosius, Christiane: India's Middle Class. New Forms of Urban Leisure, Consumption and Prosperity, Routledge, New Delhi/Abingdon 2010; Donner, Henrike (Hrsg.): Being Middle-Class in India. A Way of Life, Routledge, London/New York 2011.

40 Huntington, Samuel P.: The Clash of Civilizations and the Remaking of World Order, Simon \& Schuster, New York 1996.

41 Masters, Bruce: The Arabs of the Ottoman Empire, 1516-1918. A Social and Cultural History, Cambridge UP, Cambridge 2013, S. 195-203.

42 Dieser Glaube an die Vereinbarkeit von islamischer Religion und wissenschaftlicher Rationalität wird auch betont bei Motadel, David: Islamische Bürgerlichkeit. Das soziokulturelle Milieu der muslimischen Minderheit in Berlin 1918-1939, in: Brunner, José/Lavi, Shai (Hrsg.): Juden und Muslime in Deutschland. Recht, Religion, Identität, Wallstein, Göttingen 2009, S. 103-121. 
konnte diese Mittelklasse, die nur etwa zehn Prozent der Bevölkerung umfasste, jedoch nicht erlangen. Ab der Jahrhundertwende wurde ihr Reformeifer zudem durch das Aufkommen des arabischen Nationalismus mehr und mehr in den Hintergrund gedrängt.

Doch auch die Nationalisten in muslimischen Ländern wiesen zahlreiche Ähnlichkeiten mit den westlichen Mittelklassen auf, wie Lucie Ryzova in ihrer Studie über die ägyptische effendiyya zeigt. ${ }^{43}$ Diese urbanen Eliten verstanden sich insbesondere nach der britischen Besetzung des Landes im Jahr 1882, durch die innen der Aufstieg in der staatlichen Bürokratie erschwert wurde, als Sprecher einer unabhängigen ägyptischen Nation und sie wurden nach dem Ersten Weltkrieg zur sozial führenden Schicht. Ebenso wie Ryzova betont auch Wilson Chacko Jacob, dass die effendis keine sozioökonomische Klasse waren, sondern sich eher als kulturelle Formation verstehen ließen, die vornehmlich an der Ausbildung einer individuellen Persönlichkeit interessiert gewesen seien. Im Kern dieses Projektes habe - so Jacob die Ausbildung einer spezifisch ägyptischen Männlichkeit gestanden. Mit dieser spezifischen effendi masculinity seien die männlichen Angehörigen der Mittelschichten dem europäischen Fremdbild des degenerierten und verweichlichten Orientalen entgegengetreten. ${ }^{44}$ Auch dies kann als Hinweis auf das ambivalente Spannungsverhältnis zur europäischen Moderne gelesen werden in dem die ägyptischen Mittelschichten wie auch diejenigen in anderen Teilen der nichtwestlichen Welt standen. Einerseits war die westliche Moderne kulturelles Vorbild und anzustrebendes Ideal, andererseits stellte sie auch eine Bedrohung dar, da vieler Europäer während der Kolonialzeit - und oft auch darüber hinaus - davon überzeugt waren, nichtwestliche Eliten verblieben trotz aller Modernisierungsbemühungen gegenüber dem europäischen Vorbild stets in einer defizitären Position.

\section{Das Verhältnis zwischen Staat und Bürger in Japan und China}

Trotz der kulturellen Ähnlichkeiten zwischen außereuropäischen Mittelklassen und ihren westlichen Pendants lassen sich doch auch grundsätzliche Unterschiede feststellen. Der wichtigste ist sicherlich, dass in verschiedenen westeuropäischen Staaten und in den USA die Ideale des Bürgertums zur maßgeblichen Leitinstanz des gesellschaftlichen Ganzen wurden und zu politischen Reformen und der Einrichtung stabiler zivilgesellschaftlicher Institutionen führten. ${ }^{45}$ In großen Teilen der außereuropäischen Welt gelang dies jedoch nur teilweise. Dies gilt nicht nur für Afrika und den Nahen Osten, sondern auch für verschiedene ostasiatische Länder. Besonders erwähnenswert ist Japan, das im Zuge der Meiji-Restauration einen rasanten Modernisierungsprozess durchlief und bis Ende des 19. Jahrhunderts in wirtschaftlicher, technischer und militärischer Hinsicht zu den europäischen Mächten aufschließen konnte. Innenpolitisch war der japanische Zentralstaat jedoch weiterhin hierarchisch und autokratisch; eine Zivilgesellschaft habe sich deshalb gemäß der lange Zeit in der historischen Forschung vorherrschenden Meinung nicht herausbilden können. Diese Einschätzung ist jedoch nur teilweise korrekt. Wie ein von Gesine FoljantyJost und Momoyo Hüstebeck herausgegebener Sammelband verdeutlicht, formierten sich ab den 1870er Jahren in Japan bürgerliche Protestbewegungen, die gegen die staatliche

43 Ryzova, Lucie: The Age of the Efendiyya. Passages to Modernity in National-Colonial Egypt, Oxford UP, Oxford u. a. 2014.

44 Jacob, Wilson Chacko: Working Out Egypt. Effendi Masculinity and Subject Formation in Colonial Modernity, 1870-1940, Duke UP, Durham, NC/London 2011.

45 Osterhammel: Verwandlung (wie Anm. 1), S. 1.086.

\section{8}


Autorität ankämpften und eine konstitutionelle Monarchie und Meinungsfreiheit verlangten. ${ }^{46}$ Nach dem Scheitern dieser demokratischen Bewegung wurde 1889 die Meiji-Verfassung verkündet, die nach preußischem Vorbild entworfen worden war und die Souveränität des Tennō in den Mittelpunkt stellte. Insbesondere durch den Aufschwung nationalistischer und expansionistischer Tendenzen ab der Jahrhundertwende gerieten zivilgesellschaftliche Akteure zunehmend in die Defensive.

Drei Aufsätze des Sammelbandes eröffnen besonders interessante Perspektiven für eine globalgeschichtliche Rekalibrierung der bürgerlichen Mittelklassen: Naoshi Yamawaki zeigt in seinem Beitrag, dass politische Philosophen wie Nakae Chömin sich nicht nur durch europäische Denker beeinflussen ließen, sondern auch auf die Ideen chinesischer Philosophen wie Mengzi (dem bedeutendsten Nachfolger des Konfuzius) abstützten. ${ }^{47}$ Die Gedankenwelt japanischer Intellektueller war also nicht einfach durch eine Übernahme europäischer Ideen geprägt, sondern kann eher als Resultat eines globalen Verflechtungsprozesses gesehen werden. Hiroshi Mitani verweist in seinem Artikel zur Entstehung des japanischen Pressewesens auf die Bedeutung westlicher Vorbilder, betont aber auch die globale Zirkulation von Nachrichten und die gegenseitige Beobachtung der jeweiligen medialen Öffentlichkeit in unterschiedlichen Staaten. ${ }^{48}$ Michiko Mae schließlich argumentiert, dass in Japan Öffentlichkeit und Privatheit schwerer voneinander getrennt werden könnten als in westlichen Gesellschaften, da ab den 1890er Jahren die öffentliche Sphäre durch den Staat dominiert worden sei. Auch nach 1945 hätten sich zivilgesellschaftliche Akteure kaum als Teil einer politischen Öffentlichkeit gesehen. Dies habe sich erst in den 1970er Jahren geändert, als es neuen Protestbewegungen wie etwa der Frauenbewegung oder der Umweltbewegung gelang, eine neue Verbindung zwischen Öffentlichkeit und Privatheit herzustellen, wodurch auch Privatpersonen eine politische Initiative ermöglicht wurde. ${ }^{49}$

In China etablierte sich in den Küstenstädten ab Mitte des 19. Jahrhunderts eine neue Mittelschicht. Für chinesische Kaufleute barg die erzwungene Öffnung des Landes neue Kooperationsmöglichkeiten mit westlichen Firmen. Gleichzeitig bildete sich in treaty ports wie Shanghai eine neue intelligentsia heraus, die in Missionsschulen und an europäischen oder amerikanischen Universitäten eine westliche Bildung genossen hatte und die sich bewusst von traditionellen konfuzianistischen Ordnungsvorstellungen distanzierte. Insbesondere nach der Xinhai-Revolution von 1911, die zur Gründung der ersten chinesischen Republik führte, schien eine liberale Gesellschaft im Bereich des Möglichen. Die Wirtschaftskrise von 1923 beendete jedoch dieses "goldene Zeitalter" der chinesischen Bourgeoisie (wie es von Marie-Claire Bergère bezeichnet wurde ${ }^{50}$ ) und läutete

46 Foljanty-Jost, Gesine/Hüstebeck, Momoyo (Hrsg.): Bürger und Staat in Japan, UV Halle-Wittenberg, Halle 2013. Aufschlussreich ist dabei, dass für Philosophen wie Yoshino Yakuzo, der sich an Rousseaus Konzept der volonté générale orientierte, kein Widerspruch zwischen der Propagierung einer freiheitlichen Gesellschaft und der Unterstützung der japanischen Kolonialpolitik in Korea bestand. Dies stellte keine japanische Besonderheit dar - auch in Großbritannien unterstützten die Wortführer des Liberalismus mehrheitlich die Zivilisierungsmission des British Empire -, zeigt aber die Ambivalenz von liberalen Ideen im Zeitalter des Imperialismus.

47 Yamakawi, Naoshi: Demokratie und Civil Society in Japan aus Sicht der Public Philosopy, in: FoljantyJost/Hüstebeck: Bürger (wie Anm. 46), S. 19-40.

48 Mitani, Hiroshi: Die Formierung von Öffentlichkeit in Japan. Eine Bilanz in vergleichender Perspektive, in: ebd., S. 41-61.

49 Mae, Michiko: Auf dem Weg zu einer transnationalen und partizipatorischen Zivilgesellschaft, in: ebd., S. 177-194.

50 Bergère, Marie-Claire: The Golden Age of the Chinese Bourgeoisie, 1911-1937, Cambridge UP, Cambridge 1989 (franz. 1986). 
eine jahrzehntellange Periode der sozialen Unruhen und des Bürgerkrieges ein, die erst mit der Machtübernahme der Kommunisten im Jahr 1949 beendet wurde. Als einen der Hauptgründe für das Scheitern der liberalen Ideen nennt Yves Chevrier die Tatsache, dass die bürgerliche Mittelschicht außerhalb der kosmopolitisch geprägten Küstenstädte kaum über politischen Einfluss verfügte und es deshalb nicht schaffte, ihre Modernisierungsvorstellungen auf nationaler Ebene in ein politisches Programm umzusetzen. Diesbezüglich sieht Chevrier eine eindeutige Parallele zur postmaoistischen Umgestaltung der chinesischen Gesellschaft, die ab Ende der 1970er Jahre erfolgte. Zwar habe sich Ende des 20. Jahrhunderts wiederum eine städtische Mittelschicht etablieren können. Doch wie schon in den Jahrzehnten nach 1900 führte dies nicht zur Entstehung einer chinesischen Zivilgesellschaft. ${ }^{51}$ Dies liegt laut Jie Chen nicht zuletzt daran, dass die neue chinesische Mittelklasse ihren Aufstieg im Wesentlichen der Kommunistischen Partei verdankte und deshalb nicht an politischen Reformen und der Errichtung einer demokratischen Gesellschaft interessiert ist. In dieser Hinsicht sieht der Autor einen deutlichen Unterschied zu westlichen Gesellschaften, in denen der Durchbruch einer kapitalistischen Wirtschaftsordnung und die Etablierung einer parlamentarischen Demokratie Hand in Hand gegangen seien. ${ }^{52}$

\section{Nord- und Südamerika: Mittelklassen ohne aristokratisches, Oben'}

Anders als in Europa, Asien und Afrika mussten sich die Mittelschichten in Nord- und Südamerika nicht gegen den Machtanspruch einer alteingesessenen Aristokratie behaupten. Nicht zuletzt aus diesem Grund wurde das US-amerikanische Großbürgertum im 19. Jahrhundert nach Ansicht von Sven Beckert und Julia B. Rosenbaum zu einer der mächtigsten Wirtschaftseliten der Welt. Wie deren Sammelband zeigt, konnte sich die „American Bourgeoisie" unter anderem durch eine Teilhabe an globalen Verflechtungsprozessen von anderen Teilen der Gesellschaft - insbesondere der unteren Mittelschicht abgrenzen. ${ }^{53}$ Maureen Montgomery legt in ihrem Beitrag dar, wie diese soziale Distinktion etwa durch Reisen nach Europa und einen Bezug zur europäischen Hochkultur erfolgte. Darüber hinaus war die Zurschaustellung exotischer Kunstgegenstände - japanischen Fächern, chinesischen Vasen, Kupfertabletts aus dem Nahen Osten - bedeutsam, wie der Aufsatz von Katherine Gier zeigt. Gerade bürgerliche Frauen konnten durch eine derartige „kosmopolitische Häuslichkeit“ an der Herausbildung einer globalisierten Konsumkultur

51 Chevrier, Yves: En introduction. De la Cité problématique à la Ville habitée. Histoire et historiographie de la société urbaine chinoise au XXe siècle, in: Chevrier, Yves/Roux, Alain/Xiao-Planes, Xiaohong (Hrsg.): Citadins et citoyens dans la Chine du XXe siècle. Essais d'histoire sociale, Éditions de la Maison des Sciences de I'Homme, Paris 2010, S. 15-101.

52 Chen, Jie: A Middle Class Without Democracy. Economic Growth and the Prospects for Democratization in China, Oxford UP, Oxford u. a. 2013. Vgl. zur neuen chinesischen Mittelschicht auch Chen, Minglu/ Goodman, David S.G. (Hrsg.): Middle Class China. Identity and Behaviour, Elgar, Cheltenham/Northampton 2013.

53 Beckert, Sven/Rosenbaum, Julia B. (Hrsg.): The American Bourgeoisie. Distinction and Identity in the Nineteenth Century, Palgrave Macmillan, Basingstoke/New York 2010. Vgl. zum amerikanischen Großbürgertum auch Beckert, Sven: The Monied Metropolis. New York City and the Consolidation of the American Bourgeoisie, 1850-1896, Cambridge UP, Cambridge 2001. Für die Geschichte der amerikanischen Mittelschicht Bledstein, Burton J./Johnston, Robert D. (Hrsg.): The Middling Sorts. Explorations in the History of the American Middle Class, Routledge, New York 2001. 
teilnehmen. ${ }^{54}$ Der Band versammelt zwar aufschlussreiche und kulturhistorisch fundierte Beiträge zum amerikanischen Großbürgertum, die Geschichte der black middle class wird jedoch bedauerlicherweise nicht näher beleuchtet. Wenngleich rassistische Vorurteile auch nach dem Amerikanischen Bürgerkrieg virulent blieben, bildete sich im letzten Drittel des 19. Jahrhunderts eine afro-amerikanische Oberschicht aus. So erwirtschafteten einzelne afroamerikanische Unternehmer seit den 1870er Jahren riesige Vermögen und konnten sozial aufsteigen. ${ }^{55}$ Andere Afroamerikaner versuchten, durch Bildung und die Anlehnung an die euroamerikanische bürgerliche Kultur sowie die Mitgliedschaft in Vereinen wie der 1776 gegründeten Prince Hall Freemasonry (dem afroamerikanischen Zweig des weltweiten Freimaurerordens) soziale Disktinktion herzustellen. Da sie aber trotz aller Bemühungen bei den weißen Amerikanern auf rassistische Vorurteile trafen, näherten sie sich ab Ende des 19. Jahrhunderts wieder stärker an jene afroamerikanische Unterschicht an, von der sie sich ursprünglich hatten abgrenzen wollen. ${ }^{56}$

Gänzlich anders stellt sich die Lage in Lateinamerika dar: Obwohl hier je nach Land und Zählweise zwischen 10\% und $40 \%$ der Bevölkerung zur Mittelschicht gerechnet werden, gelang es dieser soziale Gruppe nicht, eine gesellschaftliche Machtposition einzunehmen. In nur wenigen Teilen der Erde waren - und sind - zudem die Unterschiede der Vermögensverteilung ähnlich groß wie in Lateinamerika. Diese schwache Stellung der Mittelschicht gegenüber der Oligarchie (gebildet aus Großgrundbesitzern, Minenbesitzern, Bankiers und Großkaufleuten) wird in der Forschung gemeinhin als eine der Ursachen für die fehlende gesellschaftliche Entwicklung und den virulenten Militarismus angesehen. Anders als in der europäischen Sozialgeschichte, in der eine Orientierung an geteilten bürgerlichen Werten und Verhaltensweisen als einende Klammer zwischen Großbürgertum und (unterer) Mittelschicht gilt, wird damit in den Studien zur lateinamerikanischen Geschichte die Differenz zwischen diesen beiden sozialen Gruppen betont. Als Folge der marginalen Position der Mittelschicht konnten sich weder stabile demokratische Institutionen noch eine funktionierende Zivilgesellschaft ausbilden. Mit dem Aufkommen dependenztheoretischer Ansätze wurde die Zukunft des Kontinents ab den 1960er Jahren nicht mehr in der Verwirklichung der politischen und gesellschaftlichen Utopien der Mittelklassen gesehen ${ }^{57}$, sondern in den Aktivitäten der bärtigen Revolutionäre der marxistischen Befreiungsbewegungen und in den Bauern und Arbeitermassen, in deren Namen sie kämpften. Diese Versuche, eine durch wirtschaftliché und politische Ideale der Mittelschicht geprägte Gesellschaft zu etablieren, gingen allerdings in vielen Fällen mit der Ermordung von Gewerkschaftlern, Massakern an Indios und revoltierenden Bauern sowie Militärputschen einher. ${ }^{58}$ Diese dunkle Kehrseite der ,Mittelschichtsmedaille' wird allerdings nur in den wenigsten Studien zur lateinamerikanischen Mittelklasse

54 Montgomery, Maureen E.: „Natural Distinction“. The American Bourgeois Search for Distinctive Signs in Europe, in: Beckert/Rosenbaum: American Bourgeoisie (wie Anm. 53), S. 27-44. Vgl. hierzu auch Hoganson, Kristin: Cosmopolitan Domesticity. Importing the American Dream, 1865-1920, in: The American Historical Review 107 (2002), H. 1, S. 55-83.

55 Graham, Lawrence Otis: Our Kind of People. Inside America's Black Upper Class, HarperCollins, New York 1999.

56 Muraskin, William A.: Middle-Class Blacks in a White Society. Prince Hall Freemasonry in America, California UP, Berkeley, CA u. a. 1975.

57 Vgl. etwa für die enttäuschten Hoffnungen der mexikanischen Mittelklassen und der zunehmenden Entfremdung vom regierenden Pardido Revolucionario Institucional ab Mitte der 1960er Jahre Walker, Louise E.: Waking from the Dream. Mexico's Middle Classes After 1968, Stanford UP, Stanford, CA 2013.

58 Vgl. etwa für Guatemala Grandin, Greg: The Last Colonial Massacre. Latin America in the Cold War, Chicago UP, Chicago, IL 2004. 
systematisch in die Argumentation einbezogen. David S. Parker und Louise E. Walker machen den gegenwärtigen Stand der Forschung in einem Sammelband zugänglich ${ }^{59}$, in dem verschiedene früher publizierte Aufsätze neu abgedruckt wurden. ${ }^{60}$ Der Band zeigt unter anderem, welche Rolle der ethnischen Herkunft für die Zugehörigkeit zur Mittelklasse zukam und er verdeutlicht, wie konstitutiv die Übernahme bestimmter Geschlechterkonzepte für die Ausbildung einer spezifischen Mittelklassenidentität war. Die Bedeutung derartiger Eigenschaften war jedoch je nach Zeitpunkt und Ort unterschiedlich. So war um 1950 in São Paulo eine helle Hautfarbe nötig um zur Mittelklasse gezählt zu werden, während dies in Salvador oder in Bahia nicht der Fall war. Der wirtschaftliche Aufschwung seit Mitte der 1990er Jahre hat in verschiedenen lateinamerikanischen Ländern nach einer jahrzehntelangen Stagnation zu einem erneuten Aufschwung der Mittelklassen geführt. ${ }^{61} \mathrm{Ob}$ dies zur Einrichtung einer stabilen Zivilgesellschaft führt, oder ob diese Hoffnung erneut an den internen Widersprüchen des Kontinents scheitert, bleibt jedoch abzuwarten.

\section{Europa: das vergessene Bürgertum}

Der Umstand, dass der Aufstieg des Bürgertums ausgerechnet in der Phase des Hochimperialismus erfolgte, ist den Exponenten der europäischen Sozialgeschichte nicht verborgen geblieben. Die in den späten 1960ern von Hans-Ulrich Wehler entwickelte These des Sozialimperialismus besagte, koloniale Abenteuer hätten in erster Linie den Zweck gehabt, interne soziale Spannungen abzubauen. ${ }^{62}$ Und in Großbritannien schrieb John MacKenzie gegen die Meinung an, das British Empire sei eine bloße Angelegenheit der sozialen Elite gewesen, indem er sich mit den Rückwirkungen des Imperialismus auf die britische Gesellschaft beschäftigte. ${ }^{63}$ Dabei führte auch er an, die imperiale Propaganda habe in erster Linie dazu gedient, die Klassengegensätze zu besänftigen; das koloniale Imaginarium diente somit in erster Linie der Etablierung eines ,falschen Bewusstseins'. Derartige Ansätze machen allerdings nur im Rahmen einer materialistischen Geschichtsauffassung Sinn, da sie von der Annahme ausgehen, die Bourgeoisie habe über ein eindeutiges Verständnis ihrer selbst und ihrer Interessen verfügt. Nur auf dieser Grundlage konnte sie einen ideologischen Überbau entwickeln, um ihre soziale Machtposition zu festigen. Gegen derartige Ansichten hat die kulturhistorische Bürgertumsforschung mit guten Gründen vorgebracht, das bürgerliche Selbstverständnis könne nicht einfach als Funktion einer bestimmten sozialen Position aufgefasst werden. Dieses Selbstverständnis habe sich nur im Kontext von kulturellen Codes und Repräsentationen ausbilden können, mit deren Hilfe

59 Wichtige Monographien der letzten eineinhalb Jahrzehnte waren etwa Parker, David S.: The Idea of the Middle Class. White-Collar Workers and Peruvian Society, 1900-1950, Pennsylvania State UP, University Park, PA 1998; Owensby, Brian P.: Intimate Ironies. Making Middle-Class Lives in Modern Brazil, Standford UP, Stanford, CA 1999; Barros, Fuimos Rodolfo: Aventuras y Desventuras de La Clase Media, Aguilar, Buenos Aires 2005.

60 Parker, David S./Walker, Louise E. (Hrsg.): Latin America's Middle Class. Unsettled Debates and New Histories, Lexington, Lanham, KY u. a. 2012.

61 Ferreira, Francisco H. G. u. a.: Economic Mobility and the Rise of the Latin American Middle Class, World Bank Publications, Washington 2013.

62 Siehe für Deutschland die klassische Studie von Wehler, Hans-Ulrich: Bismarck und der Imperialismus, Kiepenheuer \& Witsch, Köln 1969.

63 MacKenzie, John M.: Propaganda and Empire. The Manipulation of British Public Opinion, 1880-1960, Manchester UP, Manchester 1984.

\section{2}


sich das Bürgertum über sich und die Welt, in der es lebte, verständigt habe. ${ }^{64}$ Der koloniale Diskurs wäre gemäß einem solchen Zugang weniger Ausdruck einer bereits bestehenden bürgerlichen Hegemonie - wie die Sozialimperialismusthese postuliert hatte -, sondern eher ein Versuch der städtischen Mittelschichten, sich durch die Herstellung von Bezügen zur außereuropäischen Welt neu zu orientieren und somit auch den rasanten gesellschaftlichen Wandel des 19. Jahrhunderts zu verarbeiten.

Wie werden nun in neueren Studien die Rückwirkungen globaler Strukturen auf die europäischen Gesellschaften geschildert? Oder genauer: Welcher Stellenwert wird in diesen Untersuchungen der Verflechtung Europas mit der kolonialen Welt für die Aufrechterhaltung gesellschaftlicher Machtverhältnisse innerhalb Europas zugeschrieben? Um es vorwegzunehmen: In neueren imperialhistorisch und postkolonial geprägten Publikationen werden sozialhistorische Fragestellungen auffallend selten explizit thematisiert. Damit verschwindet das europäische Bürgertum ausgerechnet in dem Moment vom Radar der new imperial history, in dem sich die außereuropäische Geschichte mit der Entstehung von Mittelklassen in Asien, Afrika und Lateinamerika zu beschäftigen beginnt. Dies ist umso bemerkenswerter, als die Forderung, Metropole und Kolonie in einem gemeinsamen Analyserahmen zu untersuchen, ausgerechnet in einem Sammelband zur bürgerlichen Welt im imperialen Zeitalter vorgebracht wurde. ${ }^{65}$ Obwohl dieses von Ann Laura Stoler und Frederick Cooper vorgebrachte Postulat inzwischen gewissermaßen zum Mantra des Postkolonialismus wurde, herrscht bei seiner Anwendung auf die Erzeugung und Stabilisierung bestimmter gesellschaftlicher Formationen ein merkwürdiges argumentatives Vakuum. Ein neuerer von John MacKenzie herausgegebener Sammelband thematisiert zwar die "popular responses to imperialism" 66 in verschiedenen europäischen Ländern. Diese werden aber faktisch als homogene Gemeinschaften geschildert, etwa in der Einleitung des Herausgebers: „The European scramble for colonies [...] became something of a national endeavour necessarily embracing large segments of the peoples of several states, all of which were intent on securing a slice of action in the almost unstoppable project of the Europeanisation of the globe ${ }^{\prime 67}$. Inwiefern dieser von MacKenzie als „colonisation of consciousness" bezeichnete Prozess auch einen Einfluss auf die soziale Schichtung innerhalb Europas hatte, wird im Band nur am Rande thematisiert. So betont zwar Giuseppe Finaldi, der italienische Kolonialismus sei ein Versuch der Eliten gewesen, die sozialen Spannungen zu entschärfen, die aufgrund der nicht eingelösten Hoffnungen im geeinten Italien aufgekommen seien. ${ }^{68}$ Wie genau dies passierte, wird jedoch nicht geschildert. Auch die Beiträge zu Frankreich (Berny Sèbe), Belgien (Matthew G. Standard) und den Niederlanden (Vincent Kuitenbrouwer) kommen kaum über die Feststellung hinaus, das jeweilige imperiale Projekt sei durch einen Großteil der Bevölkerung unterstützt worden - wenngleich die Kolonialbegeisterung bei den Eliten am größten gewesen sei. Positiv hervorzuheben ist allerdings der Beitrag von Bernhard Gißibl, der auf Basis der

64 Sarasin, Philipp: Stadt der Bürger. Bürgerliche Macht und städtische Gesellschaft, Basel 1846-1914 Vandenhoeck \& Ruprecht, Göttingen 1997.

65 Stoler, Ann Laura/Cooper, Frederick: Between Metropole and Colony. Rethinking a Research Agenda, in: dies. (Hrsg.): Tensions of Empire. Colonial Cultures in a Bourgeoise World, California UP, Berkeley, CA u. a. 1997, S. 1-56.

66 MacKenzie, John M. (Hrsg.): European Empires and the People. Popular Responses to Imperialism in France, Britain, the Netherlands, Belgium, Germany and Italy, Manchester UP, Manchester/New York 2011.

67 MacKenzie, John: Introduction, in: MacKenzie: European Empires (wie Anm. 66), S. 1-18, hier S. 1.

68 Finaldi, Giuseppe: ,The Peasants did not Think of Africa'. Empire and the Italian's State Pursuit of Legitimacy, 1871-1945, in: ebd., S. 195-234. 
bisherigen Forschungsliteratur die Beziehungen zwischen deutschem Imperialismus und deutscher Sozialpolitik beleuchtet. ${ }^{69}$ Dabei verweist er auch auf die historiographische Pointe, dass ausgerechnet Hans-Ulrich Wehler, der seinerzeit die Sozialimperialismusthese in die deutsche Sozialgeschichte einbrachte, heute zu den dezidiertesten Kritikern der neuen Globalgeschichte und der transnationalen Geschichte gehört. ${ }^{70}$

Der These folgend, die imperiale Welt zeichne sich durch eine Dichotomie von Metropole und Peripherie aus, orientieren viele neuere Studien ihre Untersuchungen am Paradigma des Raumes und fragen danach, inwiefern sich bestimmte Nationen und Imperien in der Kolonialzeit global vernetzten. Eher beiläufig wird auf den Umstand eingegangen, dass koloniale Fremdbilder und Bezüge zur außereuropäischen Welt in vielen Fällen auf bürgerliche Akteure zurückgingen. Wie eng Sozial- und Imperialgeschichte zusammenhängen können, zeigt Andreas Zangger in seiner Studie zur "kolonialen Schweiz", wobei insbesondere die Tatsache bemerkenswert ist, dass sich derartige Bezüge auch für ein Land ohne eigenen Kolonialbesitz feststellen lassen. Zangger untersucht in seinem Buch die Netzwerke von Schweizer Kaufleuten, Plantagenbesitzern, Naturforschern und Technikern im kolonialen Südostasien. ${ }^{71}$ Auf der argumentativen Ebene orientiert sich die Untersuchung an einem methodischen Nationalismus, da nach der Verflechtung der Schweiz und der kolonialen Welt gefragt wird. Implizit ist sie jedoch sozialhistorisch angelegt, da die ausgeleuchteten - Themenfelder Handel und Wissenschaft spezifische Betätigungsfelder des Wirtschafts- beziehungsweise Bildungsbürgertums waren. Nicht die "Schweiz" knüpfte Verbindungen mit "Südostasien", sondern eine relativ genau bestimmbare soziale Gruppe - das schweizerische Bürgertum - mit ganz spezifischen Motiven war hier am Werk. So wird etwa die Entwicklung der ethnographischen Forschung als dezidiert "bürgerliche Praxis" (S. 357) bezeichnet, da sie dem Wirtschafts- und Bildungsbürgertum sowohl die Erschließung außereuropäischer Märkte erleichterte, als auch kulturelles Kapital generierte, mit dem es seine Position innerhalb der schweizerischen Gesellschaft festigen konnte. Damit wird die Erforschung der "kolonialen Schweiz" direkt anschlussfähig an die europäische Sozialgeschichte.

\section{Das Verhältnis von race und class}

Die new imperial history ist für die Sozialgeschichte insbesondere deshalb von höchstem Interesse, da sie Hinweise dafür bietet, wie sich Schichtzugehörigkeit und ethnische Herkunft - oder race und class - gegenseitig beeinflussten. Dies wird auch in der meisterhaften Studie von Catherine Hall über den Abolitionisten Zachary Macaulay und seinen Sohn, den Historiker Thomas Babington Macaulay, deutlich. Die Untersuchung zeigt, wie sich im Denken dieser beiden Männer bestimmte Vorstellungen von englischer Nation mit einer spezifischen Sichtweise auf das British Empire verband. ${ }^{72}$ Während der 1768 geborene Vater die Trennung zwischen ,Zivilisation' und ,Barbarei' noch von der

69 GiBibl, Bernhard: Imagination and Beyond. Cultures and Geographies of Imperialism in Germany, 18711945, in: ebd., S. 158-194.

70 Wehler, Hans-Ulrich: Transnationale Geschichte - der neue Königsweg historischer Forschung?, in: Budde, Gunilla/Conrad, Sebastian/Janz, Oliver (Hrsg.): Transnationale Geschichte. Themen, Tendenzen und Theorien, Vandenhoeck \& Ruprecht, Göttingen 2006, S. 161-174.

71 Zangger, Andreas: Koloniale Schweiz. Ein Stück Globalgeschichte zwischen Europa und Südostasien (1860-1930), transcript, Bielefeld 2011, S. 289-377.

72 Hall, Catherine: Macaulay and Son. Architects of Imperial Britain, Yale UP, New Haven, CT/London 2012.

\section{4}


Frage abhängig machte, ob die jeweiligen Völker christlich waren, orientierte sich das Denken von Thomas Macaulay in erster Linie an der liberalen Ideologie des Fortschrittes: England müsse als das zivilisierteste Land der Erde eine Führungsfunktion einnehmen, und die Geschicke des Landes - und damit der Welt - sollten durch die Mittelklasse gelenkt werden. So befürwortete er etwa, nur Vermögenden das Wahlrecht zuzugestehen, schließlich sei es nicht zuletzt die Institution des Eigentums, welche die zivilisierten Engländer von den "tattooed savages of the Pacific Ocean" (S. 159) unterschied, wie er Anfang der 1830er Jahre in einer Rede festhielt. Dies zeigt, wie eng das Verständnis von class und race im liberalen Denken verkoppelt war. Da Thomas Macaulay jedoch auch der Ansicht war, die Stabilität Englands hänge davon ab, gebildete Arbeiter in die nationale Gemeinschaft einzubinden, befünwortete er gesetzliche Vorschriften zur Verkürzung der Fabrikarbeitszeit. Andernfalls, so seine Überzeugung, würde die Ausbeutung der Arbeiter zur Entstehung einer proletarischen "ignoble race" (S. 194) führen. Auf imperialer Ebene wollte er von derartigen Integrationsbemühungen dagegen vorerst nichts wissen. Erst nach einer langen, vielleicht mehrere Jahrhunderte währenden Zivilisierung durch die britische Kolonialmacht wäre es denkbar, Indien durch einheimische Eliten - „brown Englishmen“ (S. 333) - regieren zu lassen. In der Karibik und in Afrika würde es vielleicht noch länger dauern, bis die dortigen Völker in der Lage sein würden, sich selber zu regieren. Die Idee, außereuropäische Länder könnten nur durch die Segnungen der englischen Kultur zivilisiert werden, bezeichnet Hall als "national/imperial conceit" (ebd.). Diese Charakterisierung greift aber insofern zu kurz, als auch andere Kolonialmächte sich zu derartigen Zivilisierungsmissionen berufen fühlten. ${ }^{73}$ Außerdem verweist der häufige Vergleich von europäischem Proletariat und Kolonialvölkern darauf, dass es Macaulay wohl weniger um die Weitergabe spezifisch englischer Werte ging, sondern viel eher um eine Erziehung gemäß den Idealen des europäischen Bürgertums.

Wie eng die Bemühungen, koloniale Völker durch die Teilhabe an einer westlich-bürgerlichen Kultur zu zivilisieren, mit der Behebung sozialer Missstände in den europäischen Großstädten gekoppelt ist, zeigen die zahlreichen Studien zum Zusammenhang von innerer und äußerer Mission. ${ }^{74}$ Auch Alexandra Przyrembels „Verbote und Geheimnisse. Das Tabu und die Genese der europäischen Moderne" untersucht diesen Zusammenhang. ${ }^{75}$ In Texten der Missionare der London Missionary Society wurden die Proletarier des europäischen Großstadtdschungels oft erstaunlich ähnlich beschrieben wie die unzivilisierten ,Wilden' in der. kolonialen Peripherie. Nicht zuletzt das Einüben eines ethnologischen Blickes in Afrika oder der Südsee, so Przyrembel, habe es den Missionaren erlaubt, die urbanen Unterschichten als Kollektiv wahrzunehmen, das - ebenso wie die kolonialen Heiden - durch eine religiöse Erziehung auf eine höhere Kulturstufe gehoben werden sollte.

73 Fischer-Tiné, Harald/Mann, Michael (Hrsg.): Colonialism as Civilizing Mission. Cultural Ideology in British India, Anthem, London 2004; Barth, Boris/Osterhammel, Jürgen (Hrsg.): Zivilisierungsmissionen. Imperiale Weltverbesserung seit dem 18. Jahrhundert, UVK, Konstanz 2005.

74 Hall, Catherine: Civilising Subjects. Metropole and Colony in the English Imagination 1830-1867, Chicago UP, Chicago, IL 2002; Comaroff, John L./Comaroff, Jean: Hausgemachte Hegemonie, in: Conrad, Sebastian/Randeria, Shalini (Hrsg.): Jenseits des Eurozentrismus. Postkoloniale Perspektiven in den Geschichts- und Kulturwissenschaften, Campus, Frankfurt a. M./New York 2002, S. 247-282; Conrad, Sebastian: „Eingeborenenpolitik" in Kolonie und Metropole. „Erziehung zur Arbeit" in Ostafrika und Ostwestfalen, in: ders./Osterhammel, Jürgen (Hrsg.): Das Kaiserreich transnational. Deutschland in der Welt 1871-1914, Vandenhoeck \& Ruprecht, Göttingen 2004, S. 107-128.

75 Przyrembel, Alexandra: Verbote und Geheimnisse. Das Tabu und die Genese der europäischen Moderne, Frankfurt a. M./New York 2011. 
Ethnische Herkunft und soziale Differenzen werden in Texten von Politikern, Sozialreformern, Missionaren, Journalisten und Ethnologen des 19. Jahrhunderts immer wieder zueinander in Beziehung gebracht. Es gibt allerdings in der aktuellen Forschung keinen Konsens darüber, wie genau die Kategorien race und class zusammenhingen und ob die eine allenfalls als Funktion der anderen aufzufassen sei. Ann Laura Stoler hat die These aufgestellt, "the cultural accoutrements of bourgeois distinction were partially shaped through contrasts forged in the [...] language of race ${ }^{\text {"76 }}$. Gemäß dieser Konzeption hat die Erfahrung ethnischer Differenzen im Zuge des Kolonialismus einen kulturellen Code bereitgestellt, mit dem auch soziale Unterschied innerhalb Europas interpretiert werden konnten. Überspitzt gesagt wird damit Klasse gewissermaßen zum Nebenwiderspruch des imperialen Rassismus erklärt. David Cannadine argumentierte dagegen in seiner Studie zur Wahrnehmung des British Empire durch die britische Aristokratie gerade umgekehrt. Er vertrat die Ansicht, dass die britischen Aristokraten die koloniale Nobilität aufgrund ihrer adeligen Herkunft als grundsätzlich gleichwertig angesehen habe und folgert daraus: „Social ranking was as important as (perhaps more important than?) colour of skin in contemplating the extrametropolitan world"77.

Anstatt eine der beiden Kategorien zu priorisieren, scheint jedoch viel dafür zu sprechen, Ethnizität und Klasse als zwei unabhängige Variablen anzusehen, die je nach Kontext einen unterschiedlichen Einfluss auf die Zuweisung sozialer Machtpositionen haben konnten. So konnte der Aufenthalt in den Kolonien für Europäer eine Möglichkeit zu sozialem Aufstieg sein - er konnte aber auch die ideologischen Grundlagen der Kolonialherrschaft in Frage stellen. So zeigt Harald Fischer-Tiné in seiner Studie zu den "weißen Subalternen" in Britisch Indien - arbeitslosen Seeleuten, Kriminellen, Landstreichern und Prostituierten -, dass die Kategorien der Kolonisierer und der Kolonisierten nicht so trennscharf voneinander geschieden waren, wie der koloniale Diskurs es suggerierte. Stattdessen mussten diese Kategorien stets durch politische Praktiken aufrechterhalten werden. Gerade die Anwesenheit von Angehörigen einer weißen Unterschicht wurde dabei als Gefahr für den imperialen Rassendiskurs angesehen. ${ }^{78}$ Umgekehrt konnte die Bedienung rassistischer Klischees für bestimmte Angehörige kolonialer Eliten auch eine Möglichkeit zu sozialem Aufstieg in Europa darstellen. Dies zeigt etwa das Beispiel des togolesischen Häuptlingssohnes John Calvett Nayo Bruce, der an einer deutschen Missionsschule eine europäische Ausbildung genoss, dann in den Dienst der deutschen Kolonialverwaltung trat und ab Ende des 19. Jahrhunderts für mehrere Jahre als Leiter einer höchst erfolgreichen Völkerschautruppe durch Europa zog und der selbstverständlich das Ziel hatte, seine Kinder an europäische Universitäten zu schicken. ${ }^{79}$ All diese Beispiele legen nahe, gegenüber den starren Dichotomien, wie sie von der postkolonialen Theorie oft vertreten werden, eine gewisse Vorsicht walten zu lassen und Klasse als Kategorie für die Analyse sozialer Beziehungen in kolonialen Regimes ernst zu nehmen.

War das Verhältnis zwischen Ethnizität und Klasse auf der Ebene der Alltagspraxis zwar höchst ambivalent, und innerhalb bestimmter Grenzen auch verhandelbar, scheint auf ideengeschichtlicher Ebene der Graben zwischen den beiden Parametern sozialer Differenzierung

76 Stoler, Ann Laura: Race and the Education of Desire. Foucault's History of Sexuality and the Colonial Order of Things, Duke UP, Durham, NC/London 1995, S. 5.

77 Cannadine, David: Ornamentalism. How the British Saw Their Empire, Lane, London 2001, S. 8.

78 Fischer-Tiné, Harald: Low and Licentious Europeans. Race, Class and ,White Subalternity' in Colonial India, Orient BlackSwan, New Delhi 2009.

79 Brändle, Rea: Nayo Bruce. Geschichte einer afrikanischen Familie in Europa, Chronos, Zürich 2007.

\section{6}


mit dem Aufkommen darwinistischer Entwicklungsideen breiter geworden zu sein. Dies zumindest ist die These eines Beitrags von Theodore Koditschek, der in einem von Catherine Hall und Keith McClelland herausgegebenen Sammelband erschien und sich mit der Bedeutung ethnischer Ungleichheit für die britische Imperialgeschichte beschäftigt. ${ }^{80}$ Koditschek untersucht darin die evolutionären Zeitvorstellungen in der britischen Geschichtsschreibung des 19. Jahrhunderts und argumentiert, durch archäologische Funde und den Durchbruch des Darwinismus habe sich ab den 1860er Jahren die Ansicht durchgesetzt, die Menschheit müsse wesentlich älter sein, als jene 6.000 Jahre, von denen die Forschung bis dahin ausgegangen war. Das führte dazu, dass Zweifel an der Ansicht aufkamen, die ,unterlegenen' kolonialen Rassen könnten innerhalb weniger Generationen zur europäischen Zivilisation aufschließen. Das Erreichen einer modernen Entwicklungsstufe wurde nun auf mehrere Jahrhunderte veranschlagt, was wiederum als Auftrag an die Europäer verstanden wurde, die weniger entwickelten Ethnien der Erde durch Kolonialisierung in die Moderne zu führen. Diese intellektuelle Umwälzung geschah pikanterweise zur selben Zeit, als den europäischen Mittelklassen durch den Aufstieg der Arbeiterbewegung eine ernsthafte interne politische Konkurrenz erwuchs. Es wäre deshalb in weiterführenden Studien zu untersuchen, inwiefern sich im bürgerlichen. Diskurs koloniale Überlegenheitsgefühle und klassenpolitische Abgrenzungsversuche gegenseitig überlagerten und beeinflussten, und wie sich diese Vorstellungen mit der Krise des Liberalismus wandelten. Madison Grant warnte etwa in seinem 1916 erschienenen Buch „The Passing of the Great Race "81 sowohl vor der sozialistischen Pöbelherrschaft wie auch vor einem globalen Rassenkrieg - wobei insbesondere der Sieg Japans im Krieg gegen Russland 1905 ein Schock für die abendländische Hybris dargestellt hatte. Dies kann als weiterer Beleg für die enge innere Beziehung angesehen werden, die Konzepte von Klasse und Ethnizität im bürgerlichen Denken des späten 19. und frühen 20. Jahrhunderts aufwiesen.

\section{Globale Netzwerke, weltweite Vergesellschaftung und die Frage nach der Weltge- sellschaft}

Eine Globalgeschichte des Bürgertums ist unweigerlich mit der Frage konfrontiert, in welcher Art von Gesellschaft sich diese Mittelklassen bewegten. Die Übertragung eines im nationalen Kontextentwickelten Gesellschaftsbegriffs auf globale Zusammenhänge istjedoch alles andere als unproblematisch. Während die Sozialgeschichte den Nationalstaat gewissermaßen als Laboratorium auffasste, in dem exemplarisch der Übergang von der Vormoderne zur Moderne beobachtet werden konnte, ist eine globale Sozialgeschichte mit dem Problem konfrontiert, den Zusammenhang von sozialen Entwicklungen in verschiedenen, sich überlagernden Räumen zu beschreiben. So implizieren die makrosoziologischen Weltgesellschaftstheorien, die Niklas Luhmann und Peter Heintz in den 1970er und 1980er Jahren entworfen haben, nicht nur, dass sich in der Neuzeit ein weltumspannender Kommunikationsraum herausgebildet habe. Diese Theorien postulieren darüber hinaus auch, dass auf globaler Ebene Strukturen entstanden, die Prozesse auf lokaler und nationaler Ebene beeinflussen, wenn nicht gar

80 Koditschek, Theodore: Narrative Time and Racial/Evolutionary Time in Nineteenth-Century British Liberal Imperial History, in: Hall, Catherine/McClelland, Keith (Hrsg.): Race, Nation and Empire. Making Histories, 1750 to the Present, Manchester UP, Manchester/New York 2010, S. 36-55.

81 Grant, Madison: The Passing of the Great Race. Or the Racial Basis of European History, Bell, New York 1916. 
determinieren. ${ }^{82}$ Eine solche Behauptung ist jedoch empirisch schwierig zu belegen, da politische Akteure in der Regel primär die Verhältnisse innerhalb des jeweiligen Nationalstaates beeinflussen wollten und erst in zweiter Linie allenfalls auch auf übernationale oder globale Strukturen Bezug nahmen. Mit dem Aufkommen kulturtheoretischer Ansätze im Allgemeinen und postkolonialer Zugänge im Besonderen wurden die theoretischen Großentwürfe der Weltgesellschaftsforschung weitgehend aus der Globalgeschichtsforschung verdrängt, da sie aufgrund ihrer funktionalistischen Argumentation, ihrer Überkomplexität und ihres inhärenten Eurozentrismus mit diesen neuen Ansätzen nicht mehr kompatibel schienen. Es ist auch nicht ganz einfach zu bestimmen, wie die in diesem Beitrag vorgestellten und größtenteils mikrohistorisch ausgerichteten Studien zur Entstehung globaler Mittelklassen in die makrosoziologischen Weltgesellschaftsentwürfe eingepasst werden könnten. Für eine geschichtswissenschaftliche Analyse ist eine Weltgesellschaft zudem auch weit schwerer greifbar als eine nationale Gesellschaft mit ihren Institutionen, Parteien und Verbänden. Zwar gibt es seit dem späten 19. Jahrhundert Versuche, international verbindliche politische und wirtschaftliche Regulierungen einzuführen. ${ }^{83}$ Und auf einer ideologischen Ebene lassen sich konkurrierende Visionen von globalen Weltordnungen festmachen. ${ }^{84}$ Doch all diese Bemühungen haben zu keiner eigentlichen Weltgesellschaft geführt, die mit jenen sozialen Formationen vergleichbar war, die innerhalb einzelner Nationen entstanden.

- Eine Lösung dieses Problems könnte sein, nicht von funktional ausdifferenzierten Gesellschaften auszugehen, sondern sich mit Vergesellschaftung in einer globalhistorischen Perspektive zu beschäftigen. Vergesellschaftung im Sinne Georg Simmels bedeutet, gesellschaftliche Ordnung nicht als etwas Statisches, sondern als etwas Prozesshaftes zu verstehen, das sich als Folge der Interaktion gesellschaftlicher Akteuren ergibt. ${ }^{85}$ Ein solcher Ansatz wäre einerseits anschlussfähig an die neuere Bürgertumsforschung, welche die Herausbildung bürgerlicher Mittelklassen als Prozess ansieht. Andererseits ließe er sich auch direkt einpassen in die aktuelle Globalgeschichtsforschung, die sich für die Bedeutung globaler Vernetzungen, und damit für soziale Interaktionen auf transnationaler Ebene interessiert. Tatsächlich lässt sich die Ausgestaltung verschiedener sozialer Sphären des 19. Jahrhunderts auch als Ergebnis von Kooperationen zwischen bürgerlichen Akteuren aus verschiedenen Teilen der Welt beschreiben. Diese konnten sowohl aus den europäischen Metropolen als auch aus der kolonialen Peripherie stammen. Die Welt des Handels etwa wurde zwar spätestens ab Mitte des 19. Jahrhunderts durch die europäische Geschäftswelt und kommerzielle hotspots wie die City of London dominiert. Außerhalb Europas kooperierten europäische und

82 Luhmann, Niklas: Die Weltgesellschaft, in: ders.: Soziologische Aufklärung, Westdeutscher Verlag, Opladen 1975, S. 51-71; Heintz, Peter: Die Weltgesellschaft im Spiegel von Ereignissen, Rüegger, Diessenhofen 1982. Vgl. für eine Diskussion dieser Ansätze Greve, Jens/Heintz, Bettina: Die „Entdeckung" der Weltgesellschaft. Entstehung und Grenzen der Weltgesellschaftstheorie (Zeitschrift für Soziologie, Sonderh. „Weltgesellschaft"), Bielefeld 2005, S. 89-119.

83 Geyer, Martin H./Paulmann, Johannes (Hrsg.): The Mechanics of Internationalism. Culture, Society, and Politics from the 1840s to the First World War, Oxford UP, Oxford u. a. 2004; Petersson, Niels P.: Anarchie und Weltrecht. Das Deutsche Reich und die Institutionen der Weltwirtschaft 1890-1930, Vandenhoeck \& Ruprecht, Göttingen 2009.

84 Conrad, Sebastian/Sachsenmaier, Dominic (Hrsg.): Competing Visions of World Order. Global Moments and Movements, 1880s-1930s, Palgrave Macmillan, Basingstoke/New York 2007.

85 Simmel, Georg: Soziologie. Untersuchungen über die Formen der Vergesellschaftung, Duncker \& Humblot, Leipzig 1908. Vgl. auch Lepsius, M. Rainer: Zur Soziologie des Bürgertums und der Bürgerlichkeit, in: Kocka, Jürgen (Hrsg.): Bürger und Bürgerlichkeit im 19. Jahrhundert, Vandenhock \& Ruprecht, Göttingen 1987, S. 79-100.

\section{8}


amerikanische Handelshäuser aber weiterhin auf Augenhöhe mit chinesischen, indischen, lateinamerikanischen und arabischen Kaufleuten. ${ }^{86}$ Diese waren sich in ihren Praktiken und ihrer kaufmännischen Kultur derart ähnlich, dass sie als Angehörige einer kosmopolitischen Bourgeoisie beschrieben werden können. ${ }^{87}$

Auch im Bereich der Wissenschaft lässt sich ab dem späten 19. Jahrhundert die Herausbildung von Netzwerken beobachten, an der bildungsbürgerliche Akteure aus verschiedenen Teilen der Welt beteiligt waren. Die Mitgliedschaft in wissenschaftlichen Gesellschaften und der Austausch von Ideen in Zeitschriften und an Konferenzen führten zur Entstehung einer globalen Gelehrtenrepublik, in der die geographische Herkunft keine Rolle spielen sollte. Wie die Beiträge in einer von Stephanie Gänger und Su Lin Lewis editierten Sondernummer der Zeitschrift "Modern Intellectual History" ${ }^{88}$ zeigen, konnten indische, osmanische, südostasiatische und lateinamerikanische Forscher um 1900 durch ein Verschmelzen europäischer Ideen mit einheimischen Traditionen eine neue intellektuelle Währung generieren, die für sich eine überregionale Geltung in Anspruch nahm. Das archäologische Wissen über die Inka entstand nicht nur in Berlin und New York, sondern ebenso in den Salons in Cuzco und die neue Disziplin der Psychologie wurde nicht nur durch Debatten in Paris und Boston geprägt, sondern auch durch solche an der Universität von Kalkutta. Allerdings klafften auch in dieser kosmopolitischen Forschungsgemeinschaft Anspruch und Wirklichkeit auseinander. Sehr oft mussten die außereuropäischen Gelehrten feststellen, dass ihnen trotz aller Anstrengungen die internationale Anerkennung verwehrt blieb. Die globale Welt der Ideen war damit ebenso durch Prozesse der Integration wie auch durch solche der Exklusion geprägt, und sie wies trotz ihrer multizentrischen Ausrichtung ein eindeutiges Hierarchiegefälle zwischen den euroamerikanischen Wissenschaftshochburgen und den Forschungslaboratorien in den asiatischen, lateinamerikanischen und osmanischen Peripherien auf.

Ähnliche Ansätze zu einer globalen Vergemeinschaftung lassen sich auch im Bereich der Politik nachzeichnen. Gerade der Widerstand gegen die Kolonialherrschaft führte zur Entstehung von "cosmopolitan thought zones", die es Intellektuellen aus verschiedenen Teilen der Welt erlaubte, ein antiimperiales Ideenrepertoire zu entwickeln. ${ }^{89}$ Kris Manjapra schildert den Wissensaustausch zwischen deutschen und indischen intellektuellen Eliten ab dem späten 19. Jahrhundert. Diese Verbindungen hätten nicht zuletzt das Ziel gehabt, Alternativen zur britisch geprägten imperialen Weltordnung zu entwickeln. Manjapra illustriert diese These àn verschiedenen Fallbeispielen, so etwa der Begegnung zwischen Meghnad Saha und Albert Einstein, der Korrespondenz zwischen Girindrasekhar Bose und Sigmund Freud und dem Versuch von Rabindranath Tagore, deutsche Wissenschaftler für eine neue indische Universität zu rekrutieren. Auch wenn die schichtspezifische Herkunft der jeweiligen Akteure und die Abgrenzung von den jeweiligen Unterschichten kaum thematisiert wird und die Bedeutung der Rivalität zwischen den europäischen Großmächten wohl zu stark betont ist - immerhin war ein Großteil der Europäer von der prinzipiellen Legitimität der Kolonialherrschaft

86 Dejung: Fäden (wie Anm. 11); Zangger: Koloniale Schweiz (wie Anm. 71), S. 41-167.

87 Jones, Charles A.: International Business in the Nineteenth Century. The Rise and Fall of a Cosmopoli$\tan$ Bourgeoisie, Wheatsheaf, Brighton 1987. Jones vertritt dabei die Ansicht, dass der kaufmännische Kosmopolitanismus Ende des 19. Jahrhunderts verschwand, da die Kaufleute mehr und mehr die Nähe zu den politischen Institutionen ihrer Herkunftsländer suchten. Diese Behauptung wird durch neuere empirische Studien allerdings nicht gestützt, vgl. Dejung: Fäden (wie Anm. 11).

88 Modern Intellectual History 10 (2013), H. 2.

89 Boehmer, Elleke: Empire, the National, and the Postcolonial, 1890-1920. Resistance in Interaction, Oxford UP, Oxford u. a. 2002; Bose, Sugata/Manjapra, Kris (Hrsg.): Cosmopolitan Thought Zones. South Asia and the Global Circulation of Ideas, Palgrave Macmillan, Basingstoke/New York 2010. 
überzeugt -, so ist die Haupterkenntnis der Studie Manjapras durchaus bemerkenswert. Europäische und südasiatische Denker konnten auch in der Kolonialzeit weitaus mehr gemeinsam haben, als lange Zeit angenommen wurde, und sie waren in der Lage, zusammen an der Verwirklichung politischer und kultureller Projekte zu arbeiten. ${ }^{90}$ Spätestens ab Ende des 19. Jahrhunderts gingen somit Angehörige der bildungsbürgerlichen Eliten in unterschiedlichen Teilen der Welt wohl davon aus, dass eine Weltgesellschaft in der Form eines „Möglichkeitsraumes" globaler Kommunikation existierte, und sie versuchten, sich an diesem transnationalen Austausch von Ideen zu beteiligen. ${ }^{91}$

\section{Die dunkle Seite der Moderne}

Wie bereits erwähnt, ist die Konzeption einerWeltgesellschaft, dieanalogzurnationalstaatlichen Gesellschaft den Ausgangspunkt von sozialhistorischen Analysen darstellen kann, nicht ganz unproblematisch. Dennoch kann es aus heuristischen Gründen sinnvoll sein, von der Existenz einer derartigen giobalen Gesellschaft auszugehen. Für die europäische Sozialgeschichte war die Untersuchung des Bürgertums untrennbar mit der Frage nach den Ursachen und Folgen sozialer Ungleichheit gekoppelt. Namentlich die Forschungsergebnisse der Arbeitergeschichte stellten eine stete Mahnung daran dar, dass der Modernisierungsprozess auch Friktionen und Verlierer produzierte. ${ }^{92}$ Und die Geschlechtergeschichte machte deutlich, dass soziale Prozesse immer auch durch - oft höchst ungleiche - Geschlechterbeziehungen begleitet und geprägt waren. ${ }^{93}$ Es wird deshalb unerlässlich sein, eine globalgeschichtliche Analyse der Mittelklassen perspektivisch in Richtung einer globalen Sozialgeschichte weiter zu denken und auf derartige Machtbeziehungen zu achten, um nicht in vorschnell in eine globalbürgerliche Behaglichkeit zu verfallen. Denn auch wenn die zahlreichen Verbindungen zwischen bürgerlichen Mittelklassen aus verschiedenen Erdteilen durchaus durch ein Streben nach Gleichheit und eine kosmopolitische Grundhaltung geprägt war, so war die moderne Welt als Ganzes doch durch markante Ungleichheiten gekennzeichnet; zwischen Mittelschicht und Unterschicht, zwischen Kolonisatoren und kolonialen Subjekten und - insbesondere innerhalb des Bürgertums - zwischen Männern und Frauen. Um diese Machtbeziehungen aufrecht zu erhalten, griff die bürgerliche Mittelschicht auch auf rassistische, sexistische

90 Manjapra, Kris: Age of Entanglement. German and Indian Intellectuals Across Empire, Harvard UP, Cambridge, MA/London 2014.

91 Für Luhmann zeichnet sich eine Weltgesellschaft primär durch die prinzipielle Möglichkeit aus, an jede Kommunikation der Welt anzuschließen, ohne dass dies durch allfällige Grenzen zwischen den jeweiligen nationalen Gesellschaften verhindert werden könnte: Luhmann: Weltgesellschaft (wie Anm. 82), S. 5355. Vgl. hierzu auch Thelen, Philip: Vergleich in der Weltgesellschaft. Zur Funktion nationaler Grenzen für die Globalisierung von Wissenschaft und Politik, trancript, Bielefeld 2011.

92 Die deutsche Sozialgeschichte hatte zudem mit der Schwierigkeit zu kämpfen, die ,Machtergreifung' des ,Dritten Reiches' mit ihren modernisierungstheoretischen Grundannahmen zu vereinen. Die daraus resultierende ,Sonderwegsthese' ging nicht zuletzt von der - inzwischen widerlegten - These aus, dass die deutsche Gesellschaft aufgrund der Schwäche des Bürgertums einen Sonderweg beschritten habe, der direkt zur ,Machtergreifung' Hitlers und zum Holocaust führte: Lorenz, Chris: „Won’t You Tell Me, Where Have All the Good Times Gone?". On the Advantages and Disadvantages of Modernization Theory for Historical Study, in: Wang, Q. Edward/Fillafer, Franz L. (Hrsg.): The Many Faces of Clio. Cross-Cultural Approaches to Historiography, Berghahn, New York/Oxford 2007, S. 104-127.

93 Vgl. hierzu Smith, Bonnie G. (Hrsg.): Women's History in Global Perspective, 3 Bde., AHA, Washington, DC 2004; Wiesner-Hanks, Merry: World History and the History of Women, Gender, Sexuality, in: Journal of World History 18 (2007), H. 1, S. 53-67. 
und generell hierarchisierende Diskurse zurück. Diese stellten gewissermaßen die dunkle Seite der Modernisierungsideologie und des bürgerlichen Strebens nach Gleichheit und Fortschritt dar. Gerade der Kolonialismus, so Frederick Cooper und Ann Laura Stoler, zeige die inhärenten Widersprüche des bürgerlichen Projektes. ${ }^{94}$ In eine ähnliche Richtung argumentiert auch Paul Gilroy in seiner Studie zum "Black Atlantic"95. Eine Globalgeschichte des Bürgertums muss sich deshalb auch mit der grundsätzlichen Frage beschäftigen, ob die Beschwörung der Mittelklasse im politischen Diskurs nicht (auch) die Funktion hatte, die Existenz einer Klassengesellschaft und das Vorhandensein globaler Ungleichheiten zu verschleiern. ${ }^{96}$ Oder wie Richard Drayton - in Bezug auf die Imperialgeschichte - meinte: "An imperial history that does not think and speak for those on the underside of global processes will be inaccurate, if not delusional, about the reality of empire, and complicit with future forms of tyranny, inequality and structural violence ${ }^{497}$.

Es könnte deshalb gewinnbringend sein, eine globalgeschichtliche Untersuchung der Mittelklassen früher oder später mit der global labour history kurzzuschließen. Diese hat inzwischen in unzähligen Studien aufgezeigt, wie die moderne Welt durch ein globales Regime von Arbeitsbeziehungen gekennzeichnet war, die sowohl freie wie auch unfreie Arbeit umfassten. ${ }^{98}$ Empirische Studien haben etwa gezeigt, welche Anstrengungen europäische Offizielle und Unternehmer nach dem Ende der Sklaverei unternahmen, um Arbeitskräfte für den Betrieb von Plantagen in kolonialen Besitztümern zu gewinnen. ${ }^{99}$ Die Entwicklung einer kapitalistischen Wirtschaft und die Kolonisierung der überseeischen Welt gingen dabei häufig Hand in Hand. Dass eine globale Sozialgeschichte, die aus einer solchen Verbindung entstehen könnte, auch die Erkenntnisse der Geschlechtergeschichte einbeziehen muss, versteht sich von selbst.

\section{Schluss}

Welchen Erkenntnisgewinn vermag eine Globalgeschichte des Bürgertums zu bieten? Erstens kann sie einer eurozentrischen Blickverengung begegnen und zeigen, dass die Herausbildung bürgerlicher Mittelschichten keine Besonderheit der westlichen Gesellschaften war; ähnliche soziale Gruppen formierten sich auch in nichtwestlichen Ländern. Der Umstand, dass auch die Herausbildung des westlichen Bürgertums als Folge globaler Verflechtungszusammenhänge verstanden werden kann, legt zweitens nahe, die Herausbildung sozialer Schichtungen nicht

94 Cooper/Stoler: Tensions (wie Anm. 65).

95 Gilroy, Paul: The Black Atlantic. Modernity and Double Consciousness, Verso, London 1993.

96 Wahrman, Dror: Imagining the Middle Class. The Political Representation of Class in Britain, c. 1780-1840, Cambridge UP, Cambridge 1995

97 Drayton, Richard: Where Does the World Historian Write From? Objectivity, Moral Conscience and the Past and Present of Imperialism, in: Journal of Contemporary History 46 (2011), H. 3, S. 671-685.

98 Drayton, Richard: The Collaboration of Labour. Slaves, Empires, and Globalizations in the Atlantic World, c. 1600-1850, in: Hopkins, A. G. (Hrsg.): Globalization in World History, Pimlico, London 2002, S. 98114; Van der Linden: Workers (wie Anm. 7); Eckert, Andreas: What is Global Labour History Good for?, in: Kocka, Jürgen (Hrsg.): Work in a Modern Society. The German Historical Experience in Comparative Perspective, Berghahn, New York/Oxford 2010, S. 169-181.

99 Beckert Sven: From Tuskegee to Togo. The Problem of Freedom in the Empire of Cotton, in: The Journal of American History 92 (2005), H. 2, S. 498-526; Zimmerman Andrew: Alabama in Africa. Booker T. Washington, the German Empire, and the Globalization of the New South, Princeton UP, Princeton, NJ 2010 . 
unabhängig von derartigen globalen Vernetzungen zu untersuchen. Drittens zeigte sich jedoch auch, dass das bürgerliche Projekt überall durch eine ambivalente Mischung aus einem utopischen Streben nach Egalität einerseits und einer strikten Abgrenzung gegen urbane und rurale Unterklassen und der Bekräftigung rassistischer und geschlechterspezifischer Machtverhältnisse gekennzeichnet war.

Neben ihrem historiographischen Potenzial besitzt die Thematik einer globalen Mittelklasse auch eine hohe politische Aktualität. Wenn heute in Medien oder politischen Debatten über die Zukunft der liberalen Weltordnung reflektiert wird, wird stets auch die Rolle von Mittelklassen in Ländern wie Russland, China, Indien, dem Iran oder Ägypten thematisiert. ${ }^{100}$ Es wird dabei allgemein vorausgesetzt, dass sich in diesen Ländern nur dann eine Zivilgesellschaft, eine leistungsfähige Marktwirtschaft und ein demokratisches Politiksystem entwickeln könnten, wenn die jeweiligen Mittelklassen an gesellschaftlicher Bedeutung gewännen. Derartige Hoffnungen auf die Etablierung einer liberalen Weltordnung stoßen jedoch in verschiedenen Teilen der Welt auf erbitterten Widerstand. In europäischen und US-amerikanischen Gesellschaften wächst die Skepsis gegenüber der globalen Durchsetzbarkeit universalistisch-westlicher Werte und gleichzeitig macht sich auch eine immer deutlichere Kritik an den Auswüchsen der Globalisierung bemerkbar, durch welche die westlichen Mittelschichten, aber auch die sozialen Unterschichten, ihre soziale Stellung bedroht sehen. ${ }^{101}$ Gleichzeitig verändert sich in verschiedenen asiatischen, afrikanischen und nahöstlichen Ländern die Haltung gegenüber dem Westen und dem westlichen Projekt der Modernisierung - wobei es nicht selten zu Konflikten zwischen lokalen Mittelklassen einerseits und der Staatmacht sowie konservativen und religiös-fundamentalistischen Teilen der Bevölkerung andererseits kommt. Eine globalgeschichtliche Analyse der Mittelklasse, besonders, wenn sie perspektivisch zu einer globalen Sozialgeschichte erweitert würde, könnte zeigen, dass diese sozialen Konflikte oft eine lange Vorgeschichte aufweisen, womit die Geschichtswissenschaft eine wichtige Orientierungsfunktion in einer immer stärker globalisierten Welt übernehmen könnte. Es ist deshalb höchste Zeit, die Frage nach der Möglichkeit - aber auch den Schwierigkeiten - der Entwicklung einer globalen Sozialgeschichte ernsthaft auf die Forschungsagenda zu nehmen.

Anschrift: PD Dr. Christof Dejung, Marie Curie Senior Research Fellow, Wolfson College, Barton Road, Cambrigde CB3 9BB, Großbritannien

E-Mail: cd513@cam.ac.uk

\section{Auswahlbibliografie}

Bergère, Marie-Claire: The Golden Age of the Chinese Bourgeoisie, 1911-1937, 356 S., Cambridge UP, Cambridge 1989 (franz. 1986).

100 Vgl. u. a. Rohwer, Jim: Asia Rising. How History's Biggest Middle Class will Change the World, Brealey, London 1995; Mead, Walter Russell/Schwenninger, Sherle (Hrsg.): The Bridge to a Global Middle Class. Development, Trade and International Finance, Kluwer, Boston, MA u. a. 2003; Davis, Diane E.: Discipline and Development. Middle Classes and Prosperity in East Asia and Latin America, Cambridge UP, Cambridge 2004; Nasr, Vali: Forces of Fortune. The Rise of the New Muslim Middle Class and What it Will Mean for Our World, Free Press, New York 2009.

101 Zunz, Olivier/Schoppa, Leonard/Hiwatari, Nobuhiro (Hrsg.): Social Contracts Under Stress. The Middle Classes of America, Europe, and Japan at the Turn of the Century, Russell Sage, New York 2002.

\section{2}


Hall, Catherine: Civilising Subjects. Metropole and Colony in the English Imagination 18301867, 556 S., Chicago UP, Chicago, IL 2002.

Jacob, Wilson Chacko: Working Out Egypt. Effendi Masculinity and Subject Formation in Colonial Modernity, 1870-1940, 440 S., Duke UP, Durham, NC/London 2011.

Jones, Charles A.: International Business in the Nineteenth Century. The Rise and Fall of a Cosmopolitan Bourgeoisie, 260 S., Wheatsheaf, Brighton 1987.

Joshi, Sanjay (Hrsg.): The Middle Class in Colonial India, 384 S., Oxford UP, New Delhi u. a. 2010.

López, A. Ricardo/Weinstein, Barbara (Hrsg.): The Making of the Middle Class. Toward a Transnational History, 464 S., Duke UP, Durham, NC/London 2012.

Parker, David S./Walker, Louise E. (Hrsg.): Latin America's Middle Classes. Unsettled Debates and New Histories, 227 S., Lexington, Lanham, KY u. a. 2012.

Pernau, Margrit: Ashraf into Middle Classes. Muslims in Nineteenth-Century Delhi, 544 S., Oxford UP, New Delhi u. a. 2013 (orig. 2008).

Ryzova, Lucie: The Age of the Efendiyya. Passages to Modernity in National-Colonial Egypt, 304 S., Oxford UP, Oxford u. a. 2014.

Stoler, Ann Laura/Cooper, Frederick (Hrsg.): Tensions of Empire. Colonial Cultures in a Bourgeoise World, 463 S., California UP, Berkeley, CA u. a. 1997.

Watenpaugh, Keith David: Being Modern in the Middle East. Revolution, Nationalism, Colonialism, and the Arab Middle Class, 308 S., Princeton UP, Princeton, NJ/Oxford 2006.

West, Michael O.: The Rise of the African Middle Class. Colonial Zimbabwe, 1898-1965, 344 S., Indiana UP, Bloomington/Indianapolis, IN 2002.

Young, Linda: Middle-Class Culture in the Nineteenth Century, 263 S., Palgrave Macmillan, Basingstoke/New York 2002. 